\title{
Elena Canadelli
}

\section{La morte di Filippo De Filippi a Hong Kong (1867). Il racconto inedito di un missionario}

Riassunto - Il saggio si concentra sulla morte dello zoologo Filippo De Filippi, avvenuta il 9 febbraio 1867 a Hong Kong durante la circumnavigazione del globo della corvetta a elica italiana Magenta. Viene proposta integralmente una lettera del 29 agosto 1867 spedita da Bernardo Viganò, il missionario del Seminario Lombardo delle Missioni Estere di Milano che assistette De Filippi nelle ultime settimane di vita, al direttore del Museo Civico di Storia Naturale di Milano, Emilio Cornalia. Questo documento, fino a oggi inedito, costituisce una preziosa testimonianza della morte di uno degli zoologi italiani più noti dell'Ottocento, tra i primi in Italia ad aderire alla teoria dell'evoluzione con la conferenza L'uomo e le scimie del 1864. L'articolo ricostruisce inoltre le polemiche e le poco note vicende testamentarie che seguirono alla morte di De Filippi. Il lavoro si serve di molti documenti inediti provenienti per la maggior parte dal carteggio di Emilio Cornalia, conservato nella Biblioteca del Museo Civico di Storia Naturale di Milano, dall'Archivio Storico dell'Università di Torino e dalla Biblioteca del Dipartimento di Biologia Animale e dell’Uomo della stessa Università.

Parole chiave: Filippo De Filippi (1814-1867), spedizione della Magenta (1865-1868), Emilio Cornalia (1824-1882), Bernardo Viganò (1837-1901), testamento, evoluzionismo.

Abstract - The death of Filippo De Filippi in Hong Kong (1867). The unpublished account of a missionary.

The essay focuses on the death of the zoologist Filippo De Filippi, who died in Hong Kong the 9 February 1867 during the sailing around the world of the Italian steam corvette Magenta. It is here presented for the first time the letter sent on 29 August 1867 by Bernardo Viganò, missionary of the Seminario Lombardo delle Missioni Estere in Milan, who took care of De Filippi in his last moments, to the director of the Museo Civico di Storia Naturale of Milan, Emilio Cornalia. This previously unpublished document represents a valuable witness of the death of one of the most well-known Italian zoologists of the Nineteenth Century. He was among the first to embrace the theory of evolution in Italy, as showed by the lecture of 1864 L'uomo e le scimie (On Man and Apes). The article also deals with the unknown testamentary controversy after De Filippi's death. Many unpublished sources are used, mostly from the historical archives of the Museo Civico di Storia Naturale of Milan (Cornalia correspondence), the University of Turin and the Library of the Animal and Human Biology Department of the same University.

Key words: Filippo De Filippi (1814-1867), the Magenta Expedition (1865-1868), Emilio Cornalia (1824-1882), Bernardo Viganò (1837-1901), will, evolutionism.

Assegnista di ricerca, Università degli Studi di Milano-Bicocca, Piazza dell'Ateneo Nuovo 1, 20126 Milano, Italia; e-mail: altariel@libero.it 


\section{De Filippi e il viaggio della Magenta}

"Ho quasi 51 anni. Ebbene: se non avessi vincoli di famiglia lascerei la mia apparentemente bella posizione e con essa la pavida Europa per la vergine natura delle savane e delle selve tropicali, la natura sverginata dei nostri paesi movendomi a nausea"1. Così scriveva lo zoologo Filippo De Filippi (1814-1867) (Fig. 1) all'amico medico Andrea Verga (1811-1895), direttore dell'Ospedale Maggiore di Milano, poco prima di riuscire a realizzare il suo sogno. Risale al 1865, infatti, la sua partecipazione in veste di responsabile scientifico alla prima circumnavigazione del globo promossa dal Regno d'Italia: il viaggio della regia corvetta a elica Magenta.

Lo scopo principale della missione consisteva nella stipulazione di accordi commerciali e diplomatici con il Giappone e con la Cina, per colmare il vuoto legislativo in cui operavano gli italiani in quelle regioni, centrali in primo luogo per il commercio della seta e del seme bachi, soprattutto in un momento di crisi della produzione serica europea, colpita dall'epidemia di pebrina ${ }^{2}$. Per la parte scientifica De Filippi volle con sé nel viaggio il giovanissimo zoologo Enrico Hillyer Giglioli (1845-1909) ${ }^{3}$ e il preparatore Clemente Biasi ${ }^{4}$. Quest'ultimo lo aveva accompagnato già nel 1862 in Persia, insieme agli zoologi Michele Lessona (1823-1894) e Giacomo Doria (18401913), durante la missione diplomatica voluta dal Governo italiano per consegnare allo Scià il collare dell'Ordine della SS. Annunziata. Il comando della Magenta fu affidato all'abile capitano di fregata originario della Savoia Vittorio Arminjon (1830$1897)^{5}$, incaricato anche di concludere i negoziati diplomatici. La spedizione fu organizzata in tempi stretti -cosa di cui non mancò di lamentarsi lo stesso De Filippi nelle lettere inviate agli amici'- sebbene già da alcuni anni ci fosse chi, come l'esploratore milanese Cristoforo Negri (1809-1896), tra i fondatori nel 1867 della Società Geo-

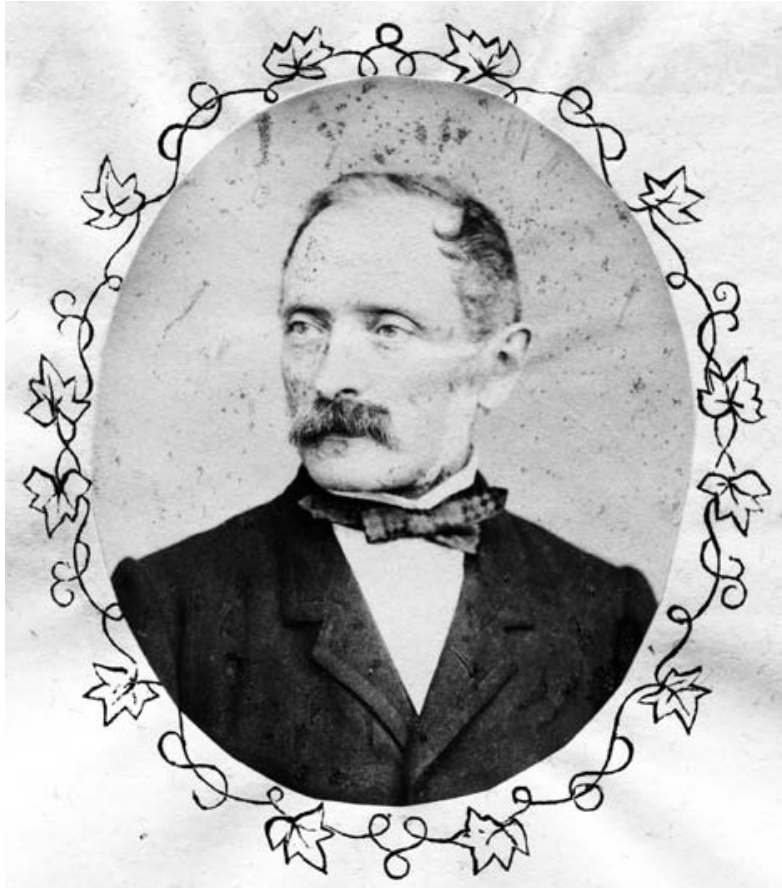

Fig. 1 - Filippo De Filippi (1814-1867). (Da Giglioli E. H., 1875). 
grafica Italiana, avesse proposto di realizzare una missione italiana in quelle terre. La decisione di partire per l'impegnativo viaggio intorno al mondo fu presa da De Filippi in meno di un mese. Rifiutata la prima proposta fattagli in ottobre dal Ministro di Agricoltura, Industria e Commercio Luigi Torelli (1810-1887) a causa della "grettezza" delle condizioni non "accettabili neppure da un principiante", una decina di giorni dopo lo zoologo rivide la sua posizione, attratto dall'idea di poter realizzare "uno dei sogni della sua vita da cui si riprometteva grandiosi risultati" . Il 19 ottobre scrisse della sua decisione a uno degli amici più cari, lo zoologo milanese Emilio Cornalia (1824-1882), domandandogli provocatoriamente: "Che dici sono matto?".

Sulle orme dei maggiori naturalisti del suo tempo, in primis l'inglese Charles Darwin che fece il giro del mondo sul Beagle, l'8 novembre 1865 De Filippi salpò con il resto dell'equipaggio (Fig. 2) sulla nave Regina da una Napoli infestata dal colera alla volta di Montevideo, dove lo attendeva la Magenta. Dal Sud America la spedizione si spinse in Estremo Oriente, seguendo la rotta di fronte al Capo di Buona Speranza; sulla via del ritorno passò dall'Australia e di nuovo dal Sud America, attraversando lo Stretto di Magellano e risalendo quindi verso l'Europa. Al contrario di Giglioli, destinato a una brillante carriera, nel marzo 1868 De Filippi non fece ritorno in patria: morì infatti a Hong Kong, a metà della spedizione, il 9 febbraio 1867, a soli 53 anni. La causa del decesso fu un'infiammazione del fegato accompagnata da dissenteria. Il medico inglese di stanza a Hong Kong John Ivor Murray (1824-1903) ${ }^{10}$ in una lettera a Giglioli del 28 aprile 1867 -da quest'ultimo pubblicata nel 1875 nel suo ampio e dettagliato resoconto Viaggio intorno al globo della R. Pirocorvetta italiana Magenta-raccontò quanto riferito dai medici che avevano assistito lo zoologo italiano negli ultimi giorni di vita: "Symptoms of abcess of the liver unmistakedly manifested themselves and ultimately the abcess burst into the intestines and the pus was voided per ano. Even then hopes of a successful result were for a time entertained, but the Senator had apparently made up

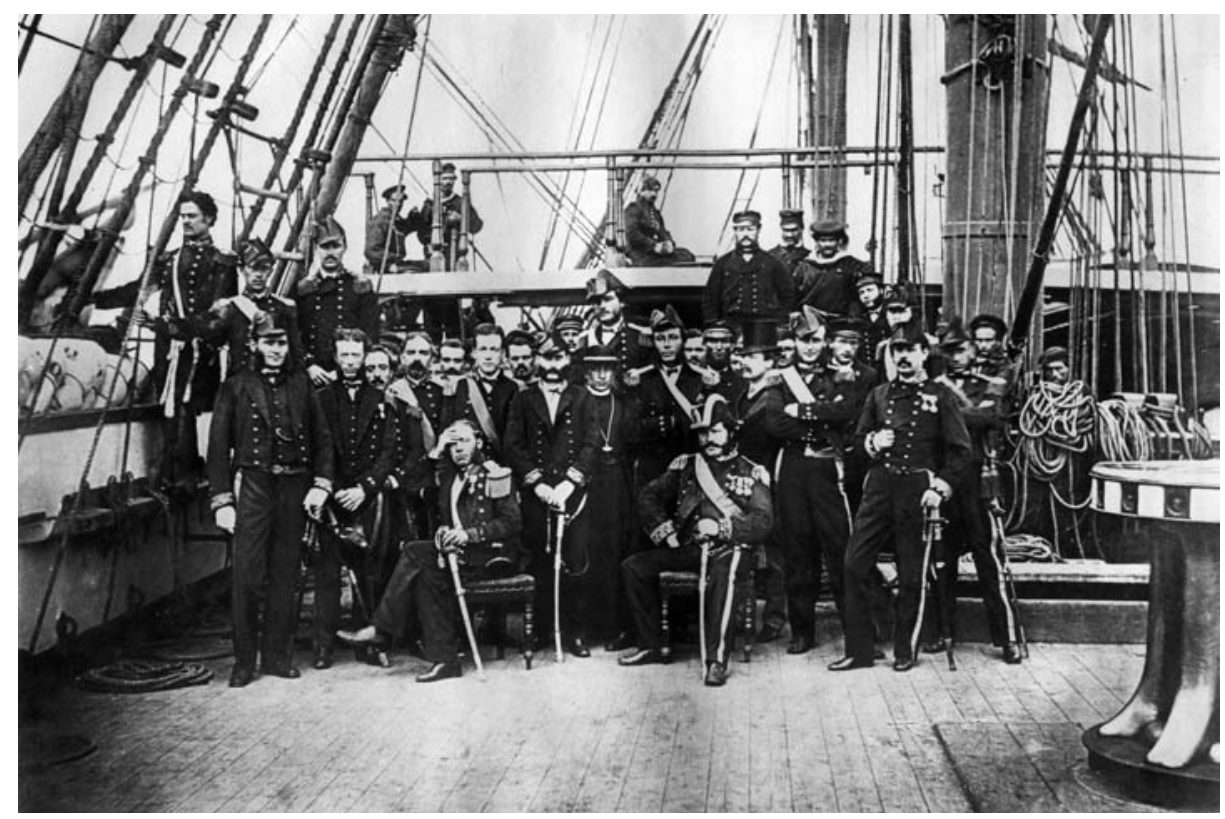

Fig. 2 - L’ultimo giorno sulla Magenta, Napoli 30 marzo 1868. (Da Giglioli E. H., 1875). 
his mind to die, and expired on the 9, of sheer exhaustion" (Giglioli E. H., 1875: 726). In base alla descrizione dei sintomi, si trattò probabilmente di un'epatite colliquativa amebica (Giacobini G. \& Panattoni G. L., 1983: 27).

All'epoca della partenza della Magenta, De Filippi era senza dubbio uno degli zoologi più noti e stimati in Italia. Nato a Milano il 20 aprile 1814, dal medico Giuseppe e da Giovanna Borsotti, De Filippi seguì le orme del padre, laureandosi in medicina all'Università di Pavia, dove ebbe come maestro il celebre anatomista Bartolomeo Panizza (1785-1867), che lasciò un segno profondo nella sua formazione. Dopo essere stato assistente di Gian Maria Zendrini (1819-1852) alla cattedra pavese di Zoologia, nel 1840 fu chiamato a Milano come aggiunto alla direzione del neonato Museo Civico di Storia Naturale. Qui il suo contributo fu fondamentale per l'organizzazione delle lezioni pubbliche e per l'allestimento del nuovo istituto, inaugurato nel 1844 in occasione della Sesta Riunione degli Scienziati Italiani. Quattro anni dopo si trasferì all'Università di Torino per insegnare Zoologia e Anatomia Comparata, cattedra rimasta vacante in seguito alla morte dello zoologo Giuseppe Genè (1800-1847). Nella città piemontese si svolse la parte più rilevante e matura della sua carriera, anche se egli rimase legato in maniera significativa al milieu scientifico e culturale lombardo della sua formazione. Nominato senatore poco prima d'imbarcarsi sulla Magenta, fu membro di prestigiose accademie scientifiche, tra cui l'Accademia delle Scienze di Torino.

$\mathrm{Al}$ di là delle numerose pubblicazioni specialistiche in diversi settori delle scienze naturali, dalla geologia alla piscicoltura, e del suo impegno editoriale nel campo della divulgazione, De Filippi è noto soprattutto per la sua adesione all'evoluzionismo. Nella famosa conferenza L'uomo e le scimie tenuta a Torino la sera dell'11 gennaio 1864, fu tra i primi in Italia ad affrontare pubblicamente il delicato tema dell'origine dell'uomo alla luce della teoria dell'evoluzione. L'inglese Thomas Henry Huxley, tra i discepoli più convinti di Darwin, si era espresso su questi temi solo un anno prima, nella nota conferenza Evidence as to Man's Place in Nature (Il posto dell'uomo nella natura). A lui, più che a Darwin -che aspettò il 1871 per dare alle stampe il suo The Descent of Man (L'Origine dell'uomo)- guardò appunto De Filippi, che si era interessato al problema dell'antichità dell'uomo già in alcuni lavori degli anni Cinquanta riguardanti i ritrovamenti di fossili umani ${ }^{11}$. Nella conferenza del 1864, pubblicata prima sulla rivista Il Politecnico e poi in volume, solide prove anatomiche e morfologiche lo portarono a rifiutare le concezioni creazioniste e fissiste tradizionali (De Filippi F., 1864). Nelle conclusioni De Filippi conciliava la tradizione religiosa con la tesi dell'origine animale dell'uomo, riservando a quest'ultimo un quarto regno separato dagli altri tre. Ciononostante, la conferenza suscitò innumerevoli critiche, tra cui quelle mossegli dal naturalista di Bologna Giovanni Giuseppe Bianconi (1809-1878), amplificate da un clima politico già particolarmente teso per la questione romana che vedeva contrapposta la Chiesa e il neonato Regno d'Italia. Fu lo stesso De Filippi a raccontare in una lettera indirizzata al suo allievo Lessona, al tempo docente a Genova, le diverse reazioni del pubblico eterogeneo presente in sala quella sera, tra cui figuravano il geologo Quintino Sella (1827-1884), il poeta Giovanni Prati, l'abate Scavia, ispettore delle scuole normali e tecniche di Torino, e l'abate Raineri, dell'Istituto dei Ciechi di Milano:

"... Ho poco tempo da spendere, tuttavia non voglio rinunciare al gusto di tracciarti alcuni gruppi dei miei ascoltatori, su relazioni genuine di testimoni auricolari. Sedevano uniti ad un banco Sella e Guerrieri e mano mano io dimostrava come in senso puro anatomico spariscano ad uno ad uno tutti i caratteri differenziali fra l'uomo e le scimmie, dicevano: bene: bravo De Filippi: ottimamente: giustissimo. Dietro di essi c'era Prati, ad ogni loro esclamazione soggiungeva: no, aspettate; vedrete; sentirete; 
conosco troppo De Filippi; le conclusioni non sono ancora giunte. Venne finalmente quel mio $M a$, nel quale aveva riposto l'effetto principale della lezione; ed allora Sella e Guerrieri ad esclamare: ahi! ahi! e Prati: udite: non ve lo aveva io detto? Bravo De Filippi. Poco disposto c'era un altro gruppo di cui facevano parte l'abate Raineri, l'abate Scavia; e lì ad ogni mia dimostrazione, smorfie colla bocca, crollatine di capo, sussulti del tronco, come rane sotto i fili di Matteucci. Venne anche per essi il mio Ma, e venne non compreso, proprio come avessi parlato turco; ma di turco non vi era che quella specie di filosofia di cui hanno pieno il cerebro questi signori"'12.

Da parte sua, anche Lessona sottolineava come il caso del maestro fosse "curiosissimo: egli ha voluto dimostrare che fra l'uomo e le scimmie sta un abisso, e il pubblico rimase persuaso dell'opposto"13. Al di là delle intenzioni di De Filippi, che non costituiscono l'oggetto principale del presente lavoro, è certo che nel 1867 il suo nome era legato a doppio filo al dibattito sull'evoluzionismo; un fatto rilevante per comprendere il modo in cui venne accolta in Italia la notizia della sua scomparsa.

\section{Il racconto della morte nel carteggio Cornalia}

Per il ruolo pubblico del personaggio, oltre che per la grande importanza diplomatica e commerciale della spedizione della Magenta, l'annuncio della morte di De Filippi a Hong Kong ricevette particolare attenzione dalla stampa e dall'opinione pubblica del tempo. La Gazzetta Ufficiale del Regno d'Italia dette la notizia il 26 marzo 1867, mentre la relazione dettagliata del viaggio fu pubblicata da Giglioli solo nel 1875. A causa della lontananza e delle difficoltà di comunicazione, le informazioni su quanto accaduto arrivarono in Italia con ritardo e furono per lo più scarse e confuse, come dimostra una differente versione dei fatti riportata da Carlo Cattaneo (1801-1869) in una lettera del 30 marzo 1867 alla moglie Anna Woodcock. Si tratta al momento dell'unica testimonianza che si distanzia dal resoconto ufficiale e che attribuisce la causa della morte di De Filippi a un ferimento avvenuto sulla Magenta durante una tempesta, nel tratto tra Montevideo e l'Isola di Giava. Cattaneo, con cui il giovane De Filippi aveva collaborato negli anni milanesi per la stesura delle Notizie naturali e civili su la Lombardia (1844), riferiva di uno strano incontro avuto durante il suo viaggio a Firenze:

“... Starting from Milan, I found myself vis-à-vis with a lady in black, who addressed me crying, and told me a very sad story. She was M.e Vallardi, the mother of poor M.e De Filippi. She had just received the sorrowful tidings of Doctor De Filippi's death in China. He was going to Japan with the ship of war Magenta. The ship took fire; with the greatest efforts it was saved; soon after they han a dreadful storm; De Filippi got a heavy blow from a piece of timber in his chest; it broke one of his ribs; and gave a dangerous contusion to his liver. He was brought back to a town on the coast of China. The idea of having lost the scientifical purpose of his journey broke his heart. In three weeks he died. M.e Vallardi was going to Bologna to see De Filippi's daugther, who his [is] married there to professor Richiardi of the university"14.

La donna in lutto diretta a Bologna era la suocera di De Filippi, Angela Ostinelli, vedova del libraio Giuseppe Vallardi (dell'Antica Ditta di Pietro e Giuseppe Vallardi di Milano) e madre di Angela Vallardi. La figlia dell'editore milanese, con cui lo zoologo pubblicò ad esempio il suo manuale di zoologia "ad uso della gioventù italiana" Regno animale (1852), aveva sposato De Filippi a Milano nel $1842^{15}$, lasciandolo ben presto vedovo. Dal matrimonio era nata una sola figlia, Elisa, che all'epoca dei fatti narrati da Cattaneo abitava a Bologna, dove il marito, Sebastiano Richiardi (1834-1904), insegnava Zoologia e Anatomia Comparata all'Università. Al momento è difficile stabilire la fonte 
di queste informazioni, dovute forse alla concitazione di quei giorni, forse a un errato collegamento tra avvenimenti realmente accaduti. La notizia dell'incendio scoppiato nel cuore della notte del 24 aprile 1866 sulla Magenta e prontamente domato dal comandante e dagli ufficiali, infatti, era vera. Ne parlavano sia Giglioli nella sua relazione sia De Filippi in una lettera del 16 maggio 1866 a Lessona (Lessona M., 1867: 656; Giglioli E. H., 1875: 114). Come realmente accaduta era anche la violenta tempesta che aveva sorpreso la Magenta il 12 febbraio 1866, una decina di giorni dopo aver lasciato la foce del Rio de la Plata. In quell'occasione erano rimasti contusi alcuni membri dell'equipaggio, tra cui Giglioli, che si era slogato la mano destra, e lo stesso De Filippi, che cadde "contro la scala che conduceva in coperta, staccandosi la cartilagine di una costola"16.

Oltre ai medici di stanza a Hong Kong, pochi furono in grado di raccontare le ultime settimane di vita dello zoologo. Nessun membro dell'equipaggio della Magenta, infatti, assistette in prima persona all'evento, apprendendo della morte del compagno solo il 12 marzo 1867 durante una sosta a Giava. Sebbene incompleta, la testimonianza del giovane Giglioli rimane comunque centrale per ricostruire i giorni trascorsi dalla spedizione italiana nella colonia britannica prima del tragico episodio. Dopo aver concluso con successo gli accordi diplomatici e commerciali con il Giappone e la Cina, la Magenta approdò a Hong Kong (Fig. 3) il 19 dicembre 1866. Ai primi di gennaio De Filippi partì per un'escursione a Macao insieme al luo-

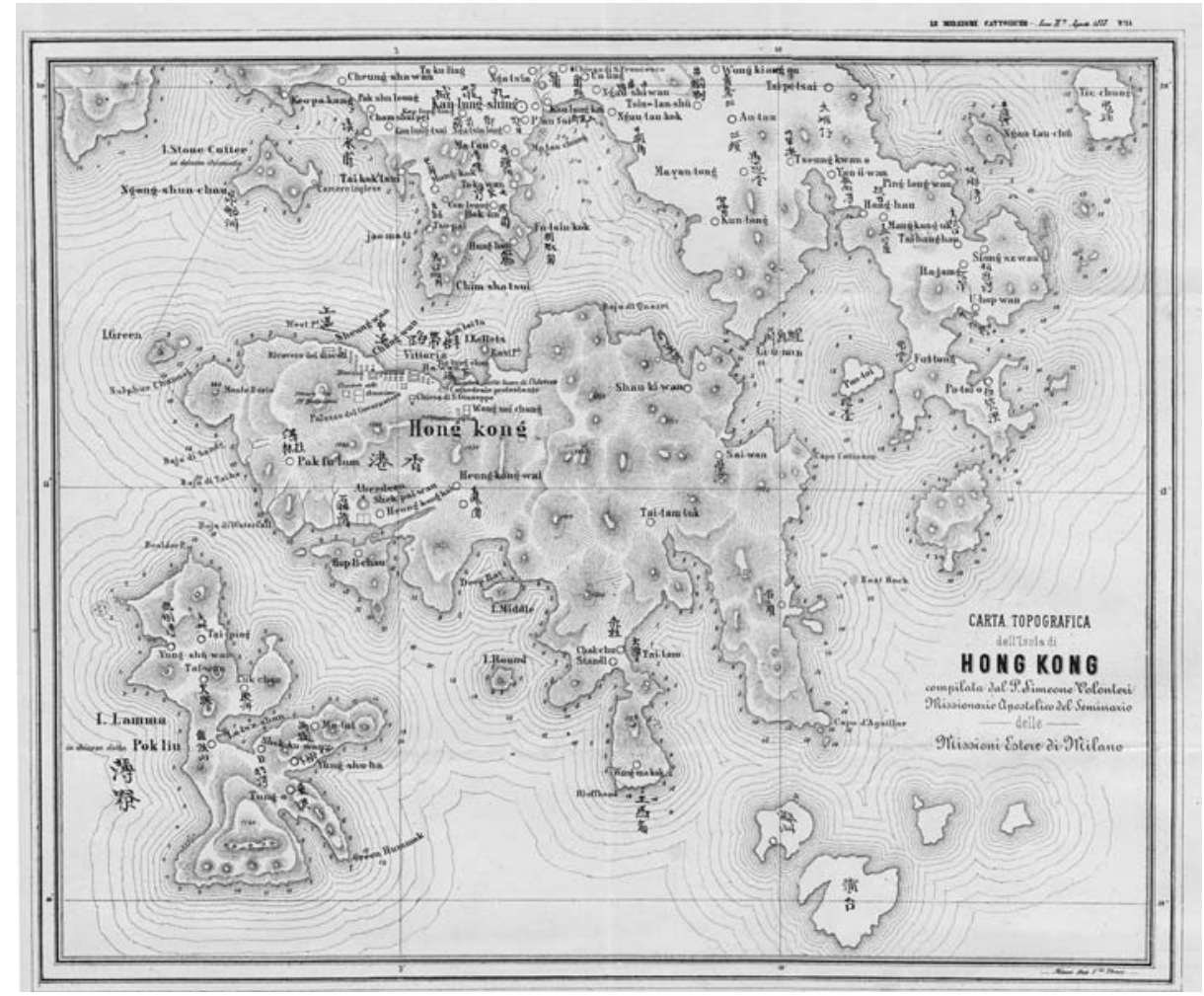

Fig. 3 - Carta topografica di Hong Kong compilata dal missionario del Seminario Lombardo delle Missioni Estere di Milano, Simeone Volonteri (1831-1904), e terminata nel 1867. (Da Le Missioni Cattoliche, n. 35, agosto 1873). 
gotenente di vascello Cesare Sanfelice e a Filippo Canevaro, che a Macao risiedeva. Ritornato a Hong Kong il 9 gennaio con i primi sintomi della malattia, si aggravò in breve tempo, obbligando la nave a rimandare la partenza. Il 18 gennaio, dopo essersi consultato con i medici, il comandante Arminjon decise di salpare per Singapore, ma fu costretto a ritornare dopo due giorni a causa del peggioramento delle condizioni di salute dello zoologo ${ }^{17}$. Una volta sbarcato De Filippi, il 26 gennaio la pirocorvetta fece rotta verso l'Australia. Egli fu lasciato alle cure dell'inglese John Dent (1822-1892 $)^{18}$, console italiano a Hong Kong, ma soprattutto a quelle di Bernardo Viganò (1837-1901) ${ }^{19}$ (Fig. 4), sacerdote del Seminario Lombardo delle Missioni Estere (attuale Pontificio Istituto Missioni Estere, PIME), che in quella missione era appena arrivato nel 1865, rimanendovi poi per ben 35 anni.

De Filippi morì dopo poche settimane, il 9 febbraio. Fu sepolto nel cimitero cattolico della Happy Valley, in una tomba di 25 piedi inglesi ${ }^{20}$, contrassegnata da una semplice lapide con l'anno di morte. Solo nel 1872 il commerciante in seta Vincenzo Comi (?-1896), tornando in Italia dopo un lungo soggiorno in Giappone, dove aveva contribuito tra l'altro alla buona riuscita dei negoziati tra Vittorio Arminjon e le autorità giapponesi, sostituì alla croce di legno una lastra di marmo con epitaffio ${ }^{21}$. Dopo numerosi tentativi falliti, le spoglie dello zoologo rientrarono in Italia nel settembre 1879. Giunte a Venezia sulla Ceylon, furono trasportate a Pisa e deposte con una cerimonia pubblica nel Camposanto della città toscana. Qui nel frattempo si era trasferita la figlia Elisa con il marito Richiardi, nominato professore di Zoologia e Anatomia Comparata in quella Università.

Bernardo Viganò, il cui nome compariva anche sulla Gazzetta Ufficiale del 26 marzo 1867, ebbe un ruolo da protagonista nelle vicende legate alla tragica scomparsa

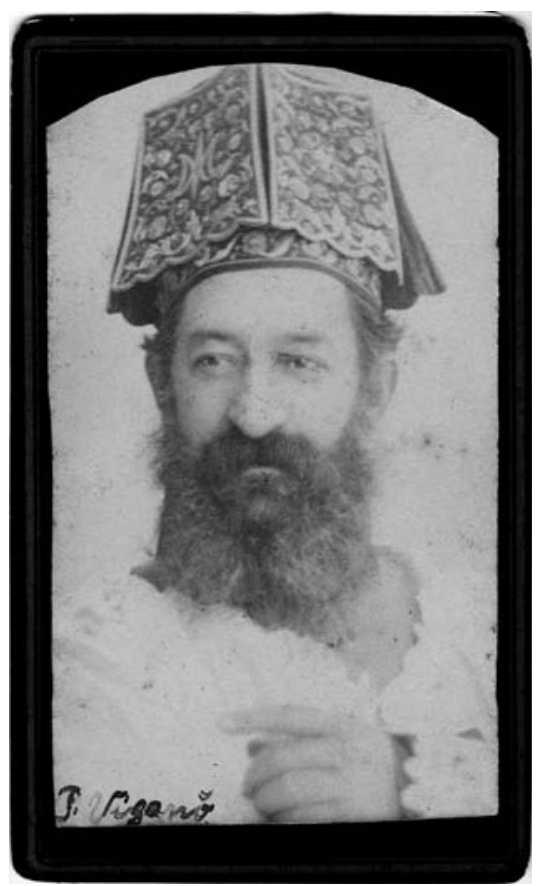

Fig. 4 - Bernardo Viganò (1837-1901). (Da Archivio Fotografico PIME, Milano). 
di De Filippi, in un momento di difficoltà economica della missione e di passaggio di consegne tra l'anziano e da tempo malato sacerdote veronese Luigi Ambrosi (18191867), morto solo un mese dopo lo zoologo, e il suo successore Giovanni Timoleone Raimondi (1827-1894), fratello del noto esploratore del Perù, Antonio Raimondi. Fu Viganò a scrivere sotto dettatura il testamento, a dargli l'estrema unzione, a spedire in Italia il telegramma del 14 febbraio con la notizia del decesso, a colloquiare con alcuni membri della sua famiglia, a occuparsi della sepoltura, a chiedere istruzioni per il rientro della salma o per l'erezione di un monumento in suo onore. È proprio grazie a una lunga lettera del 29 agosto 1867 spedita dal missionario originario di Monticello, vicino Lecco, a Emilio Cornalia, all'epoca direttore del Museo Civico di Storia Naturale di Milano, che si possono seguire in presa diretta gli avvenimenti di quei giorni, colmando il vuoto narrativo lasciato da Giglioli.

Questo significativo documento, fino a oggi inedito e di seguito trascritto integralmente, è conservato nel carteggio Cornalia depositato presso la Biblioteca del Museo. La lettera non si trova insieme alle altre di Viganò nel fascicolo a lui intestato, ma in un altro con le lettere di varie personalità che in quegli anni operavano a diverso titolo in Estremo Oriente: da quelle del primo console italiano in Giappone, il milanese Cristoforo Robecchi (1821-1891), a quelle del setaiolo piemontese Pietro Savio (1838-1904) o della contessa Mathilde Ruinart, moglie di Vittorio Sallier de La Tour (1827-1894), primo rappresentante del Regno d'Italia a Yokohama ${ }^{22}$. Probabilmente Cornalia contattò Viganò su richiesta del genero di De Filippi, lo zoologo Sebastiano Richiardi. Questi, infatti, non appena appresa la notizia della morte, aveva scritto al naturalista milanese, raccontandogli di non essere soddisfatto delle scarse notizie fornitegli dal console italiano a Hong Kong, anche in merito alla delicata questione dell'eredità ${ }^{23}$. Richiardi chiese a Cornalia di interpellare Viganò, che sapeva aver assistito il defunto, per ringraziarlo di quanto aveva fatto e per avere una lunga lettera di chiarificazione, scritta per l'appunto dal missionario il 29 agosto.

Il direttore del Museo Civico di Storia Naturale e Viganò rimasero in contatto almeno fino al 1869, anche durante le peregrinazioni del missionario nel resto della Cina e nelle Filippine, soprattutto per questioni concernenti i bachi da seta, materia che interessava molto Cornalia, autore dell'importante Monografia del bombice del gelso (1856) e sensibile alle questioni bacologiche in un momento di crisi di uno dei settori più redditizi della Lombardia. Il legame tra queste due figure, apparentemente distanti, va letto alla luce degli stretti rapporti esistenti all'epoca a Milano tra il Seminario Lombardo delle Missioni Estere (detto anche Seminario di S. Calocero) e il Museo Civico di Storia Naturale. Lo stesso Viganò nella parte finale della sua lettera a Cornalia ricordava con nostalgia le visite giovanili in Museo. Nel 1863 il Seminario, istituito nel 1850 e a lungo diretto da monsignor Giuseppe Marinoni (1810-1891), aveva donato al Comune di Milano la sua collezione etnografica, dando vita al primo e più significativo nucleo della Raccolta etnografica del Museo Civico. Dai missionari in Estremo Oriente giungevano inoltre bachi da seta, carte geografiche, notizie, oggetti etnografici e reperti antropologici e naturalistici (Livi P., 2008: 279-281). A tenere le fila di questi proficui rapporti fu soprattutto Cornalia, in contatto con un gran numero di viaggiatori in ogni parte del mondo, tra cui molti esuli lombardi espatriati dopo il 1848.

Insieme ai documenti provenienti dall'Archivio Storico dell'Università di Torino e dalla Biblioteca del Dipartimento di Biologia Animale e dell'Uomo della stessa Università, il carteggio Cornalia rappresenta oggi una fonte rilevante per ricostruire gli eventi di quel lontano $1867^{24}$. Accanto a Michele Lessona -amico, collaboratore e poi successore di De Filippi alla cattedra di Zoologia e Anatomia Comparata di Torino- 
grazie ai suoi contatti, alla sua posizione e ai suoi interessi, Cornalia ebbe un ruolo di primo piano nella vicenda che seguì alla morte di De Filippi. Egli fu al centro di un fitto scambio e "smistamento" di notizie tra gli eredi De Filippi e i viaggiatori italiani che in quegli anni si trovavano tra Cina e Giappone, per lo più religiosi, diplomatici e commercianti in seta, come l'imprenditore serico di Milano Ferdinando Meazza (1838-1913) che fece da intermediario tra Cornalia e Viganò durante i suoi frequenti viaggi in Giappone ${ }^{25}$. Per avere informazioni sulla scomparsa dell'amico, si rivolsero a Cornalia anche molti colleghi zoologi. Tra questi, il milanese Paolo Panceri (18331877), trasferitosi a Napoli nel 1866 per insegnare Anatomia Comparata all’Università partenopea, gli scrisse "col cuore pieno di amarezza per la perdita del nostro carissimo De Filippi, [...] onde temperare un poco il dolore che mi ha accagionato, col parlarne un poco tra di noi che lo avevamo tanto in pregio e che gli volevamo tanto bene", pregandolo di "scrivermene quanto più puoi poiché tu sei nelle occasioni di avere avuto altri dettagli altre notizie" 26 . Anche gli scienziati torinesi contattarono Cornalia per un aiuto nella stesura delle loro commemorazioni: da Lessona, che in primavera ricordò l'amico in una conferenza nell'Anfiteatro di Chimica presso S. Francesco di Paola a Torino $^{27}$ e in una biografia pubblicata sulla popolare rivista Nuova Antologia, al fisiologo di origini olandesi Jakob Moleschott (1822-1893), che lo ricordò nella prestigiosa sede dell'Accademia delle Scienze di Torino ${ }^{28}$.

L'amicizia tra Cornalia e De Filippi era di lunga data, come raccontò lo stesso direttore del Museo di Storia Naturale nella commossa commemorazione Intorno alla vita, e ai lavori scientifici di Filippo de' Filippi tenuta a Vicenza nel corso della Terza Riunione Straordinaria della Società Italiana di Scienze Naturali (14-17 settembre 1868) ${ }^{29}$. Il discorso rimase inedito, ma gli appunti preparatori sono oggi conservati nelle carte di Cornalia presso il Museo Civico. Da questo importante documento emerge un ritratto umano, oltre che scientifico, di un personaggio amante della caccia, del disegno, della musica e della poesia di Dante e del Porta, di un naturalista appassionato, che "men che poteva stava nel suo gabinetto", preferendo "i boschi, i laghi, i campi, e i monti" 30 . A testimoniare oggi questo profondo legame personale e professionale è anche la corrispondenza scambiata tra i due, circa 150 lettere datate dall'inizio degli anni Quaranta fino alle ultime spedite durante la navigazione della Magenta, da Rio de Janeiro, Montevideo, Saigon, Yokohama, Pechino, fino all'ultima del 29 dicembre 1866 da Hong Kong. In questa, a circa un mese dalla morte, De Filippi definiva ottima la sua salute, sebbene aggiungesse che "la gloria di compiere il giro del mondo" gli pesava "per mille ragioni" 31 . Le lettere spedite a Cornalia durante la navigazione sono ricche di osservazioni naturalistiche ed etnografiche sui luoghi visitati, oltre che di commenti sugli avvenimenti politici italiani e sulla spedizione, che per la parte scientifica era stata a suo dire "montata da cane"32. Vi si trovano inoltre preziose informazioni sulla vita a bordo, sulla fervente attività di raccolta del materiale durante le escursioni a terra, sullo stato delle trattative diplomatiche e sul rapporto con il giovane Giglioli, caldamente raccomandato a Cornalia, nonostante qualche piccola critica.

\section{Le polemiche italiane: la morte di un evoluzionista}

Come ben mostra la lettera di Bernardo Viganò a Cornalia, riportata in calce a questo articolo, i religiosi italiani presenti a Hong Kong ebbero un ruolo centrale nel diffondere informazioni sulla scomparsa di quello che in Italia era considerato uno dei maggiori sostenitori del darwinismo ${ }^{33}$. Il 26 marzo 1867, lo stesso giorno della Gazzetta Ufficiale, l'Osservatore Cattolico pubblicò uno stralcio del 
telegramma spedito il 14 febbraio da Viganò alla redazione del giornale. Il missionario specificava che prima di morire, De Filippi aveva chiesto e ottenuto due volte i sacramenti. Alla notizia della sua riconciliazione con la Chiesa Cattolica, seguiva un commento sferzante della redazione, che metteva in relazione la morte del naturalista con la sua adesione all'evoluzionismo:

“... I nostri lettori saranno desiderosi di sapere se questo onorevole senatore sia quel medesimo professore, che trasse in scena la famosa teoria della nostra provenienza dalle scimie. Lo è infatti, ma ci si assicura che egli per un fenomeno un po' strano non credeva di andar contro né alla fede cattolica, né alla storia mosaica, e ci si afferma, che rispettava religiosamente le sante scritture, ed era un buon cristiano, un buon cattolico. Certi cervelli non v'è che il Signore che li possa penetrare. Noi riprovando altamente quella stranissima e umiliantissima dottrina, che ci fa bestia un po' più grossa della bertuccia, ci consoliamo però pensando, che il sig. De Filippi non fosse in cattiva fede, e che abbia chiusi i suoi giorni con un attestato sincero della sua piena unione alla santa Chiesa cattolica" (Morte del senatore De Filippi, 1867).

Più neutro rispetto all'Osservatore Cattolico fu invece il Museo delle Missioni Cattoliche di Torino, che pubblicò integralmente una lettera del 15 febbraio spedita da un altro religioso italiano che a Hong Kong ebbe modo d'incontrare De Filippi: Luigi Moccagatta da Castellazzo (1809-1891), vicario apostolico delle province dello Shantung e dello Shanxi, da lungo tempo in Cina. Insieme ai missionari del Seminario Lombardo, il religioso piemontese aveva accolto la Magenta al suo arrivo nel porto della colonia britannica. Sulla nave aveva trascorso un breve periodo, durante il quale aveva approfondito la conoscenza di De Filippi. Come Viganò, Moccagatta ricordava la religiosità dimostrata dallo zoologo negli ultimi giorni della sua vita: dalla confessione e protesta di fede cattolica davanti agli ufficiali della Magenta all'aggravarsi della malattia, quando "sino agli ultimi momenti tutto compunto da sé baciava il Crocifisso, e tenendolo in mano tranquillamente se ne mori" (Moccagatta L., 1867: 275). La stessa Gazzetta Ufficiale, del resto, non trascurò di menzionare i "conforti della religione" richiesti e ricevuti "con fervore" da De Filippi.

A questa versione dei fatti si oppose il fronte anticlericale, nelle cui fila rientravano i collaboratori della rivista Il Libero Pensiero. Giornale dei Razionalisti, da poco fondato a Milano dall'intellettuale democratico Luigi Stefanoni (1842-1905). Nell'arco di qualche mese la rivista ospitò una polemica tutta italiana riguardante la religiosità manifestata da De Filippi in punto di morte; discussioni che oggi possono apparire irrilevanti, ma che non lo erano affatto in un clima che vedeva fortemente contrapposti clericali e anticlericali. Il 4 aprile in un breve articolo in ricordo dello zoologo, dal titolo significativo Non è possibile, lo scrittore e giornalista Mauro Macchi (18181880) contestava energicamente il resoconto ufficiale, scagliandosi contro i "corvi chinesi" che si erano precipitati "addosso al De Filippi quand'egli era già fatto cadavere, $\mathrm{o}$, per lo meno, aveva già perduto l'uso delle sue forze e de' suoi sensi" (Macchi M., 1867: 222). Le polemiche che investirono De Filippi in seguito alla conferenza sull'uomo e le scimmie del 1864 non lo abbandonarono dunque nemmeno dopo la morte. Egli continuava a dividere l'opinione pubblica, nonostante si fosse preoccupato di conciliare le nuove teorie biologiche con la tradizione religiosa.

Al dibattito prese parte anche l'amico Lessona, che nell'estesa biografia pubblicata sulla Nuova Antologia criticò sia chi aveva proclamato De Filippi "campione di materialismo" sia chi aveva visto in lui un nemico della Chiesa. La strumentalizzazione che della sua morte fecero sia il fronte cattolico più intransigente sia quello degli anticlericali del Libero Pensiero era a suo parere inaccettabile: "Tanto il gior- 
nalista quanto i predicatori, invero, non conoscevano il De Filippi; per poco che l'avessero conosciuto, non solo non avrebbero fatto le meraviglie della sua morte confortata dalla religione, ma sarebbero stati persuasissimi che la cosa non poteva essere altrimenti" 34 . A Lessona rispose qualche mese dopo il direttore della rivista, Luigi Stefanoni, che lo accusò di aver fornito un'immagine falsa dello zoologo scomparso, presentato come un qualsiasi "meschino bigotto", confondendo così un vago senso di religiosità con la "religione del sillabo e dell'inquisizione, della confessione e del purgatorio" (Stefanoni L., 1868: 22). Quest'ultima non poteva che essere estranea a De Filippi, a cui andava il merito di aver aperto il dibattito pubblico italiano sulla teoria dell'evoluzione. Quelle intorno alla sua adesione all'ortodossia cattolica non furono le uniche polemiche che accompagnarono la morte di De Filippi. Altre, di carattere più privato, riguardarono infatti la sua eredità.

\section{La contrastata vicenda dell'eredità De Filippi}

De Filippi dettò le sue ultime volontà al missionario Bernardo Viganò il 2 febbraio 1867, una settimana prima di morire. Una copia del testamento è oggi conservata presso l'Archivio Storico dell'Università di Torino, insieme a numerose carte che permettono di ricostruire le complicate vicende testamentarie. Lo zoologo dichiarò che tutte le disposizioni erano state "concepite a mente perfettamente chiara" e "da me attentamente lette e in tutto approvate e di nuovo rilette alla presenza del $\mathrm{R}$. Console Italiano Sig. Dent all'istante della apposizione della mia firma e del sigillo consolare". De Filippi lasciò le poche cose di sua proprietà all'unica erede, la figlia Elisa, nata a Milano nel $1843^{35}$. I vestiti e gli oggetti personali che aveva con sé, compresa l'uniforme e due fucili da caccia, andarono a Viganò, "perché possa soccorrere qualche disgraziato italiano in Hong Kong"36. Una parte di questi effetti personali fu destinata a Fulvio Piacenza ${ }^{37}$, un giovane originario di Milano, che lo assistette durante la malattia e che aiutò poi a imballare i materiali della Magenta in cambio di un dollaro al giorno. A lui De Filippi lasciò anche la somma necessaria per fare ritorno in Italia, dove poté effettivamente raccontare a Cornalia gli avvenimenti di quei giorni ${ }^{38}$. Le spese sostenute dallo zoologo in quel breve periodo furono coperte da un fondo di 500 sterline depositate a Parigi e da una discreta somma di denaro consegnata al gestore dell'Hotel d'Europe, dove alloggiò nelle sue ultime settimane di vita, e che in caso di sua morte dovevano essere spedite alla figlia.

Per quanto riguardava $\mathrm{i}$ "moltissimi oggetti d'industria cinesi e giapponesi" raccolti per conto del Ministero di Agricoltura, Industria e Commercio e destinati al Regio Museo Industriale Italiano di Torino fondato nel 1862, quattro casse rimasero sulla Magenta, mentre il resto del materiale fu sbarcato a Hong Kong insieme a De Filippi. Nel corso del viaggio lo zoologo aveva acquistato per suo conto numerosi oggetti, tra cui cinque rotoli di seta, lacche, bronzi e porcellane, che solo lui, come scrisse nel testamento, era in grado di separare dal resto del materiale. Per questo, in caso di morte, egli proponeva al Ministero di acquistare in blocco tutti gli oggetti al prezzo di favore di 1000 lire da corrispondere alla figlia. De Filippi non dimenticò però l'Istituto a lungo diretto, il Museo Zoologico dell'Università di Torino, a cui lasciò la sua ricca biblioteca scientifica. Al medesimo Museo andavano restituiti anche il microscopio, alcuni libri e la piccola collezione di animali sotto alcool, mentre tutto il resto doveva essere recapitato al Ministero di Agricoltura, Industria e Commercio.

Questi, dunque, furono gli ultimi desiderata di De Filippi, resi noti per sommi capi anche sulla Gazzetta Ufficiale del 26 marzo 1867. Come emerge soprattutto 
dalla documentazione conservata all'Archivio Storico dell'Università di Torino, tuttavia, le cose andarono in parte diversamente. La famiglia infatti non accolse favorevolmente le disposizioni testamentarie dello zoologo, appellandosi di volta in volta a diverse irregolarità del testamento. Più della figlia Elisa, a essere deluso fu il genero Sebastiano Richiardi, che pare "si tenesse certo della dote e che sia stato gravemente disilluso!!"39, come riferiva Paolo Panceri a Cornalia, dopo aver parlato brevemente con Michele Lessona, di passaggio per Napoli, ventilando la presenza di dissapori tra i coniugi Richiardi. Già poco dopo aver appreso della morte del suocero, Richiardi aveva scritto a Cornalia una lettera risentita, in cui a proposito dell'eredità diceva di sentirsi defraudato dei suoi diritti, ferito per la mancanza di fiducia da parte di De Filippi, di cui era stato per un periodo assistente a Torino, "mal compensato del contegno che sempre tenni con lui" "40. In attesa di ricevere maggiori chiarimenti, Richiardi attendeva "colla febbre l'arrivo delle casse" da Hong Kong, ma la sua delusione non fece che aumentare quando il Ministero della Pubblica Istruzione gli inviò una copia integrale del testamento, da cui emergeva chiaramente che "tutti i suoi libri, memorie, monografie, la collezione di animali sotto spirito che aveva fatto durante il viaggio, ogni cosa, tutto" 41 era destinato al Museo Zoologico. Ad aggravare il malcontento di Richiardi si aggiunse poi la notizia che alla cattedra di Zoologia e Anatomia Comparata, diversamente da quanto egli si aspettava, era stato nominato Lessona, che al Ministero godeva tra l'altro dell'influente appoggio di Quintino Sella ${ }^{42}$. "Converrai", scriveva nuovamente a Cornalia, "che ciò è troppo. Io non ho la virtù di reggere. Ho dato la mia dimissione non voglio più servire un governo che calpesta riguardi, diritti, leggi, io avevo sul Lessona il diritto dell'anzianità, la legge prescriveva il concorso. Il De Filippi legava al Museo di Torino l'amico. Cosa che potesse arrecare alla sua famiglia un vantaggio altri ne fruirà. Io non ho il dovere di sopportare i falli di tutti. Le conseguenze della mia deliberazione saranno gravi gravissime ma anche alla pazienza vi è un limite ed è stato fatalmente oltrepassato" "43.

Verso metà aprile tra gli eredi e l'Università di Torino, rappresentata proprio dal "rivale" Lessona, iniziarono le trattative che si conclusero due anni dopo. Il Ministero maggiormente coinvolto fu quello della Pubblica Istruzione. Se, dopo varie contrattazioni sul prezzo e sulla restituzione di somme di denaro alla famiglia ${ }^{44}$, gli oggetti e le collezioni andarono come previsto al Museo Industriale e a quello Zoologico, la ricca biblioteca, collocata negli appartamenti di De Filippi nel Palazzo dei Reali Musei di Torino, ebbe invece un destino diverso. All'inizio di maggio Elisa Richiardi De Filippi accettò l'eredità del padre con beneficio d'inventario, rendendo così obbligatoria la stima dei beni ${ }^{45}$. L'8 e il 9 maggio si procedette all'inventario dei mobili alla presenza dell'ornitologo Tommaso Salvadori (1835-1923), assistente del Museo Zoologico, di un cancelliere del Tribunale, di un perito legale e di un procuratore dell'erede. Alla fine del mese fu stabilito il valore della biblioteca, che rappresentava la parte più consistente dell'eredità. Il libraio Ermanno Loescher presentò una stima sommaria dei libri, da cui si apprende che la biblioteca era composta da 50 volumi di miscellanee rilegate e da 30 ancora da rilegare, del valore approssimativo di 1500 lire; da opere scientifiche in più volumi, del valore di 500 lire; e da miscellanee meno pregiate e di argomento vario, del valore di 500 lire, per un totale commerciale di 2500 lire, che secondo il libraio poteva salire fino a 6000 nel caso in cui gli acquirenti fossero stati istituti scientifici o collezionisti ${ }^{46}$.

Nel frattempo, da uno stato delle attività e delle passività presentato dagli eredi al Ministero della Pubblica Istruzione risultò che i debiti superavano il valore dell'asse ereditario. Per questo la famiglia De Filippi chiese all'Università di Torino 
di rinunciare al legato dei libri. Nel settembre 1867 l'allora Ministro Michele Coppino (1822-1901) rispose positivamente a queste richieste, "considerando come il Legato suddetto venga a restare senza effetto, essendo la eredità assorbita totalmente dai creditori, i quali trattandosi di eredità accettata col beneficio dell'inventario, debbono essere per i primi soddisfatti" e "volendo pur usare riguardo alla famiglia dell'illustre trapassato, che fu tanto benemerito della scienza"47. Un anno dopo aver rinunciato alla sua parte di eredità, l'Università di Torino avviò nuove trattative con gli eredi, finalizzate questa volta ad acquistare la biblioteca, in particolare i 50 volumi di miscellanee per le quali offerse 1200 lire. Lessona si spese in prima persona per la riuscita dell'iniziativa, che non andò però a buon fine. Nel corso delle contrattazioni, infatti, la famiglia cambiò idea e decise di vendere solo una parte dei volumi; condizione che non venne accettata dal nuovo Ministro della Pubblica Istruzione, Emilio Broglio (1814-1892), il quale giudicò indecoroso da parte del Governo accettare un "collezione incompiuta e di minor valore". Il 9 gennaio 1869 il Ministro chiese al Rettore di Torino di "tentare un ultimo ufficio presso la figliuola del medesimo professore; facendole sentire che il Ministero consentì di buon grado alla spesa convenuta di L. 1200, perché la predetta collezione rimanesse come uno dei ricordi del compianto De Filippi; ma che intendeva che la medesima fosse completa, alla quale condizione attribuiva un valore particolare". L'erede, aggiungeva, "non tralascerà di considerare quanta sconvenienza vi sarebbe nello smembrare una raccolta che evidentemente quell'uomo insigne voleva che formasse di per sé una serie svariata di lavori scientifici riuniti assieme come testimonianza della sua diligenza e del suo amore per la scienza che coltivava"48. Pochi mesi dopo, la risposta del Rettore al Ministro non lasciò spazio a ulteriori contrattazioni: gli eredi avevano venduto ad altri la biblioteca, l'affare era dunque saltato ${ }^{49}$. In effetti, in quegli stessi giorni Richiardi informò Cornalia di aver venduto a un non precisato acquirente "la raccolta delle monografie possedute dal De Filippi rilegate in 50 volumi al prezzo di $£ 1000$ ", commentando: "Non è un gran prezzo ma discreto, vi era del buono ma anche qualche poco di Marocco". Secondo la sua versione dei fatti, "le aveva prima proposte al Museo di Torino che non le volle acquistare. Otto giorni dopo che le avevo spedite a Napoli, il Lessona ne fece premurosa ricerca". Il Direttore del Museo Zoologico, spiegava Richiardi, aveva sperato "di averle al prezzo che le piaceva, si è ingannato e pentito"

Parallelamente alle vicende della biblioteca, grazie ai documenti torinesi è possibile ricostruire anche la sorte toccata ai preziosi taccuini, appunti e disegni realizzati da De Filippi durante il viaggio della Magenta. Questi materiali giunsero da Hong Kong nelle dodici casse piene di oggetti menzionate dallo zoologo nel suo testamento. Arrivate nel febbraio 1868 al Ministero degli Esteri nell'allora capitale Firenze, le casse furono dapprima consegnate al Ministero di Grazia e Giustizia e quindi inviate a Torino, per essere aperte il 17 marzo 1868 nei locali del Museo Zoologico, a mezzogiorno in punto, alla presenza delle autorità giudiziarie competenti, di Lessona, in rappresentanza del Ministero della Pubblica Istruzione, dell'ingegnere Giovanni Codazza (1816-1877), vicedirettore del Museo Industriale (a nome del Ministero di Agricoltura, Industria e Commercio), e dell'erede Sebastiano Richiardii ${ }^{51}$ Per l'incompletezza dei documenti oggi a disposizione, non è ancora chiaro cosa successe quel giorno. Quel che è certo è che Richiardi entrò in possesso degli importanti manoscritti, suscitando il disappunto del Governo, che considerava quei materiali di sua proprietà, trattandosi di "una parte di lavoro del capo di una missione scientifica ordinata dal Governo, e a spese di questo compiuta, anziché uno studio privato" 52 . 
Pasquale Villari, il Ministro della Pubblica Istruzione allora in carica, avviò le pratiche per riavere i taccuini nel marzo 1869, un anno dopo l'apertura delle casse, quando Lessona lo informò "in via ufficiosa" che durante il viaggio De Filippi "fece diversi disegni e scrisse alcuni appunti sopra piccoli taccuini i quali ora sono nelle mani della famiglia" ${ }^{53}$. Questi manoscritti servivano infatti al giovane Giglioli, l'unico in grado di decifrarli, che nel frattempo, grazie all'appoggio di Lessona, aveva ricevuto dal Governo l'incarico di studiare i reperti raccolti dalla Magenta e di pubblicare la relazione scientifica del viaggio ${ }^{54}$. Gli eredi rifiutarono l'offerta di 400 lire avanzata per il loro acquisto dal Direttore del Museo Zoologico di Torino, ancora una volta incaricato delle trattative, sostenendo di avere ricevuto un'offerta più vantaggiosa. Richiardi confidò probabilmente in un rilancio da parte del Ministro, che però non arrivò. I taccuini rimasero quindi alla famiglia. Di fronte all'ennesimo rifiuto di Richiardi, che negò perfino il prestito dei documenti dietro "pecuniaria ricognizione", il 13 settembre 1869 un Villari spazientito chiedeva al Rettore di Torino chiarimenti su cosa fosse realmente accaduto quel 17 marzo 1868: "I rappresentanti delle parti interessate che assistettero all'apertura delle casse contenenti gli oggetti portati in Italia dalla Magenta, avvertirono l'esistenza degli appunti e dei disegni? Di questi fu fatta consegna al Richiardi d'accordo fra le parti interessate? $\mathrm{E}$ in caso affermativo per quali ragioni le parti stesse s'indussero a tale consegna? E della consegna risulta da qualche verbale od atto qualsiasi steso dalle parti?"55. Purtroppo le risposte di Lessona, Codazza e del pretore Battisti, che sappiamo essere state spedite al Ministero della Pubblica Istruzione nell'ottobre $1869^{56}$, non sono conservate né all'Archivio Centrale dello Stato né nel fascicolo riguardante la successione De Filippi presso l'Archivio Storico dell'Università di Torino.

Il gesto di Richiardi, a cui il Ministro lasciò comunque "la piena libertà di pubblicare quelle notizie che credesse raccogliere sul viaggio scientifico del proprio suocero" 57 , fu ben presto noto tra la comunità degli zoologi italiani, che dalla spedizione della Magenta si aspettava importanti risultati. Preoccupati, ne discutevano privatamente tra loro Cornalia e Panceri, che da Napoli domandava al Direttore del Museo Civico di Storia Naturale di Milano se il giornale di De Filippi sarebbe mai stato pubblicato ${ }^{58}$. Nel settembre 1868, in occasione della Riunione dei Naturalisti Italiani di Vicenza, lo stesso Cornalia chiuse la sua commemorazione facendo voti che gli ultimi lavori dell'amico De Filippi non andassero perduti, fiducioso che "qualche naturalista in possesso dei materiali raccolti colmerà la lacuna"s9. Il riferimento a Richiardi, intervenuto alla Riunione, era esplicito. Le preoccupazioni del resto erano giustificate. Ancora nel 1870 il geografo Cristoforo Negri, sodale di De Filippi che aveva seguito da vicino la spedizione della Magenta, nel Bollettino della Società Geografica Italiana tornò sulla restituzione dei manoscritti, che sarebbero stati più utili a Giglioli, al lavoro in quegli anni per terminare la relazione scientifica del viaggio, pubblicata nel 1875: "Vorremmo che i numerosi disegni lasciati dal compianto De Filippi non mancassero a sì importante pubblicazione, e solo il Professor Giglioli suo compagno di viaggio, avendoli studiati con lui, può illustrarli. Che ne è però di questi disegni? Quali pratiche sono state fatte per riaverli? Le nostre glorie scientifiche sono poche e dobbiamo tenercele care" (Negri C., 1870: 259). Nominato nel 1871 professore di Zoologia e Anatomia Comparata all'Università di Pisa, dove intraprese una brillante carriera che lo portò anche a essere Rettore, Richiardi non pubblicò mai i manoscritti del suocero, contenenti "una quantità di cose che sono affatto inintelleggibili ed indecifrabili" 
Delle osservazioni e impressioni di De Filippi sulla missione della Magenta rimangono le tre dettagliate lettere a carattere scientifico pubblicate negli Atti dell' Accademia delle Scienze di Torino ${ }^{61}$, di cui l'ultima risale al 10 gennaio 1867, insieme ai carteggi più privati con gli amici e collaboratori italiani, come Cornalia, Negri e Lessona, che pubblicò alcuni stralci delle lettere speditegli da De Filippi durante il viaggio nella biografia apparsa sulla Nuova Antologia ${ }^{62}$. Se non fosse morto, forse De Filippi avrebbe davvero pubblicato le sue impressioni di viaggio una volta tornato in Italia, come aveva scritto a Negri dal Mar Giallo nel novembre 1866, rifiutando l'offerta di proporre qualche contributo a un giornale italiano: "Non mi sento in grado di far dello stile, fra il continuo mutar di scena nel nostro rapido e finora felicissimo viaggio. Metto a registro materiali pel caso che io possa trovare in Italia la necessaria quiete per svilupparli e dar loro un po' di forma" ${ }^{63}$. La morte a Hong Kong gli impedì di portare a termine questo proposito. La lettera del missionario Bernardo Viganò qui trascritta ci consegna una preziosa testimonianza, fino a oggi sconosciuta, degli ultimi momenti di vita di uno degli zoologi più noti del suo tempo.

\section{Un documento centrale: la lettera di Bernardo Viganò}

\section{Egregio Signore}

Hong Kong li 29 Agosto 1867

Giustizia vuole che prontamente Le dirigga una risposta, almeno quale mi è permessa, alla pregiatissima di Lei in data 3 Aprile 1867. Da un mese mi accorsi che alcune delle mie lettere non furono ricapitate e non mi è stupore giacché troppo vi è da lamentare sul nostro Servizio Postale di costì, e mi accorsi per una lettera dell'Onorevolis.a Signora Nina De Tavel ${ }^{64}$. Allora diressi, or fanno due mesi circa un pronto riscontro alla signora medesima per soddisfare alle più giuste brame dell'addolorata parentela ed amici. Ella Sig.re Direttore certamente avrà di già avuto sifatta comunicazione mentre pregava la Sig.ra De Tavel a rimediare sì all'avvenuto disordine come alla strettezza di tempo concessami. Però ricevuta dal Giappone a mezzo Postale la di Lei direttami dal Sig.r Meazza Ferdinando ${ }^{65}$ che non avea potuto consegnarmela di presenza per la confusione del trasbordo non voglio perdere la prima partenza del Piroscafo per uno schiarimento invero necessario. Ella già conosce a fondo l'attività, il zelo indefesso illimitato del estinto Senatore, il quale per virtù si sacrificò avanti tempo. L'idea di condurre infine la propria Missione servì non poco a precipitare la malattia a morte, poiché la Piro Corvetta Magenta ricevuto ordine di partire per l'Australia sostava due giorni, indi salpava nell'intenzione di passare a Singapore a tutta macchina perché l'On.e Senatore così avea espresso desiderio di tentare una prova, tanto più che i medici della Marina sì Inglese che Americana nulla trovavano di pericoloso nella seria infermità. A questa consulta anche il pensiero d'una temerarietà nella prova scomparve dalla mente dell'Infermo ${ }^{66}$. Io lo salutai allora ad Ancora levata tenendo mal soffocato il dolore di saperlo in sì grave stato e così ardito, però confortavami non poco di vederlo fra Amici e Compatrioti generosi che lo veneravano. Passarono 24 Ore di Agitazione quando scorgo alla montagna segnalata una Corvetta Italiana, miro curioso a distanza se vi fosse sopra segnale di morte e non v'era allora riavutomi da quello spavento, ma non quieto corsi diffilato a bordo, per saperne novità, ed era il Senatore aggrava ${ }^{67}$. Che vuole Direttore stimabilis. anche a questo punto parlare all'Infermo di Sbarco era un fuor d'opera; aspettiamo, così mi diceva, qui all'Ancora posso rimettermi in due o tre giorni, lo farei e 
l'avrei fatto se nulla avessi d'attendere, ma ho molto da lavorare (sic). Che dovea io fare mentre 1'Eccl.o Comandante ${ }^{68}$ se lo vedeva morire a Bordo e incolpando se stesso d'avere aderito al tentativo della partenza, piangeva come ragazzo, il Dottore l'Ottimo Sig.r Cucca ${ }^{69}$ insisteva per un provvedimento di disimbarco intanto che era possibile, l'Egregio Prof. Giglioli tentava ogni maniera di persuasione unitamente al Dottore, ma tutti restammo del pari muti. Fu solo con altra Consulta di più Medici ${ }^{70}$ che si poté indurre al domani, dubbio invero per il tempo, se non che quando Dio volle, concertai per l'oggi e per il momento che era calma, ma a questi patti sine qua non impostimi dall'Infermo che Prof. Giglioli e Preparatore Biasi continuassero il viaggio e la Missione sino a che Egli potesse raggiungerli od in Australia o quanto meno in America. Altro ostacolo e grave mentre s'era stabilito per il disimbarco d'ambedue, ed il Prof. Giglioli col Biasi avevano già preparato i Bauli; difficoltà fu questa abbastanza grave mentre i due Compagni troppo mal soffrivano di abbandonare il loro Capo ed in tale stato ${ }^{71}$. Il Governatore di qui ${ }^{72}$ voleva alloggiarlo, la nostra casa della Missione era preparata, ma l'Ammalato volle appartamento libero per non dare incommodi a chicchessia, fece quasi violenza il Console nostro di qui l'On. Sig.r Dent $^{73}$, tutto inutile ed il Medico insisteva perché al sopravvenire d'un forte vento, egli non avrebbe permesso, sicché io m'affrettai correre in cerca di una conveniente dimora e disporre per un servizio quale l'esigeva l'On.e De Filippi e quale me l'ebbe imposto il Comandante. L'alloggio fu all'Albergo dell'Europa ${ }^{74}$, e per servente si ebbe un Milanese ex Ufficiale in America, che ora trovasi a Milano al N. 4 Contrada dell'Annunziata di nome Fulvio Piacenza. Prima che disbarcasse il Cav.e Arminjon elesse una Commissione di Bordo per esaminarne la convenienza, approvato il tutto si sbarcò $^{75}$. Sig.r Direttore non vale la pena a descrivere quel momento solenne, il cupo silenzio di tutti interrotto dalla fiocca voce del povero Senatore, la generale commozione, lo scambio d'un breve saluto cogli Uffiziali, le parole d'incoraggiamento e d'esortazione ai Soldati serrò a tutti il fiato. Avea portato la mia lettiga a Bordo, ve lo accomodammo e così passò al suo letto. Il giovine Piacenza non l'abbandonò un momento per tutto il restante tempo, ed io il solo tempo per la Messa e la visita all'Ospitale a cui dovea trovarmi alle $8 \frac{1}{2}$ ant.e per la visita essendo qui disbarcati due soldati Napoletani per etisia ${ }^{76}$, più la piccola relazione a S. Ecc. il Governatore di qui che quotidianamente voleva essere informato d'ogni andamento. La speranza che sino all'Agonia del Compianto Senatore viveami in petto di poterlo restituire ai Parenti, agli Amici, alla Patria ed alle Scienze non mi permetteva di staccarmi da quel letto, anche per diffenderlo dalle molte visite che l'avrebbero disturbato e sacrificato con maggior precipizio, per cui di visita non avea che il Console gentilissimo invero ed affettuosissimo. Casa, Cucina, Mandre tutto mise a disposizione dell'Infermo, quanto supponeva potesse abbisognare e quanto desiderò l'Infermo, lo si deve all'attenzione del Sig.r Dent, dal quale m'ebbi ordine per maggior prontezza di servizio, di diriggermi al Maggiordomo Credenziere della Casa. Della malattia dirò, come dopo tre giorni cessasse la terribile dissenteria e pareva meno molestato né suoi sonni, che non ebbe mai veramente quieti, sì che svegliandosi sospirava e continuamente esponevami non sapere l'origine de' suoi tristi sogni, sempre lamentava di vedersi così indebolito da non potere raggiungere i suoi Compagni e non compire l'incominciata impresa, vi erano però dei momenti di calma ed in questi mi dettò l'atto di sua ultima volontà e rovvistò le carte distruggendone alcune le altre segnando a destinazione $\mathrm{o}$ pel Museo Tecnologico di Torino ${ }^{77}$ o pel Ministero ${ }^{78}$, siccome fu di tutte le altre cose delle quali tutte feci N. 10 Casse pel Ministero e due per detto Museo. Nel penultimo giorno sostenuto con serviziali di Brodo trovò Egli medesimo miglioramento, 
entrava in stanza ed Egli mi diresse la parola chiara e robusta: Guarda Viganò che ci mettiamo una pezza. L'ultimo giorno i Medici ${ }^{79}$ notarono anch'essi un ravvivamento, era calmo sereno e destatosi dopo due ore di sonno (erano le 3 ore dopo $1 / 2$ notte) volle che andassi a casa a dormire, (la Missione era di contro) dissemi: Buon riposo io vi darò un altro colpo. Dopo due ore fui chiamato d'altro moribondo e mi distaccai alle 7 circa per correre e vederlo agonizzante. Al giorno 1 febbraio dovendo partire il Postale chiesi se voleva scrivere qualche cosa per Italia, mi disse: no, scriverò che corro a casa per finire la convalescenza; e ridendo, se avrò a morire preparerò le cose in regola. Questo Le aggiungo a quanto avrà sentito dalla Sig.ra De Tavel ${ }^{80}$, alla quale pur dissi dei Funerali ${ }^{81}$ e come l'abbia assicurato provvedendo il terreno. Me ne duole che il Sig.r Console Dent sia obbligato per i suoi privati interessi recarsi a Londra, però ripeto che qualora si volessero le Ceneri dell'estinto Senatore, mi sarà onore prestare anche quest'opera, dispongano pure sopra di me con tutta la libertà per e fin dove credono bene. Eccomi all'ordine da sincero amico.

Qualche parolina anche sul Museo di cui Ella è degnis. Direttore. Ella certamente invitandomi con tanta gentilezza a rimetterle qualche oggetto non si sarà posto in attesa di tanti, e ne sono motivi la condizione del viaggiatore a piedi che è tutta propria del Missionario in questa parte vero vagabondo, più l'idea un po' superficiale ed una vera ignoranza in siffatta materia non mai coltivata anzi quasi non veduta se si eccettui qualche vaga lettura di curiosità, le occupazioni ecc, a mio credere se dai Missionarj poco si spedisce è certo per il timore di fare spedizioni inutili di oggetti inconcludenti. Io sorpasserei senza presunzione al mio rossore quando Ella fossemi gentile di compatimento, l'elezione che degnossi Ella fare della nostra piccola raccolta basta a far premuroso ogni Missionario per arrichirla, il Rev.do D. Aless. Ripamonti ${ }^{82}$ di costì amante assai delle cose cittadine facevami sempre calde istanze, nelle molte volte che visitammo cotesto Museo insisteva perché non s'avesse a dir chiuso a nostro riguardo, ed io mentre ringrazio infinitamente Lei Sig.r Direttore per avere messo al pubblico quei pochi oggetti e per l'affezione che conserva ai poveri esuli Milanesi Missionarj, interamente abbandonato nella sua benignità e colla più ingenua confidenza di quando in quando Le spedirò il mio piccolo doveroso tributo, e continuamente starò alla vedetta per impossessarmi d'alcun che, il quale sarà a manifestarle quella riconoscenza e quella simpatia che Ella si merita a diritto col suo zelo. Vidi che nel Museo vi erano dei teschi e parmi alcuno in gesso ${ }^{83}$, e se non erro di Cinesi non ve n'era alcuno, parmi non inutile lo spedirne un pajo appena mi sarà fattibile dovendo io stesso essicarli, tale concetto l'esposi anche al nostro Compianto Sen.e De Filippi quando assieme visitammo gli Ospitali ed i luoghi di pena, luoghi di mia giurisdizione, solo nel mese scorso il Dottore in $\mathrm{Capo}^{84} \mathrm{mi}$ favoriva N. 4 Teschi $^{85}$ pel museo di Torino, desiderati dal Senatore. Esso mi mostrò pure l'ultima lettera ricevuta dal Museo e precisamente da Lei Sig.r Direttore ${ }^{86}$, nella quale Ella instava per non essere dimenticato, poi raccomandavasi non so per quali oggetti in particolare di cui non mi ricordo, e nel mostrarmela mi diceva: Siamo Milanesi e colle nostre affezioni stiamo uniti attorno al Duomo. Non mi sarà cosa più cara che quella di soddisfare fin dove posso arrivare alla intenzione dell'Illustre nostro Compatriota Def. Il Sig.r Meazza venuto alla mia stanza vidde dei prodotti calcarei della Canapa, delle Sementi così raccolte per fare qualche confronto od esperimento, dissemi che anch'esse non sono inutili. Questo poco spedirò se altro non mi capiterà fra le ugne, Le spedirò pure un'abbastanza grossa e graziosa Pelle Torpidorus Concincinensis ${ }^{87}$ (così trovai classificato in un Opuscolo d'ogni miscela su queste coste) duolmi che sia la sola pelle, i marinaj discesi per riparazioni sulle 
Coste della Concincina ${ }^{88}$ sulle roccie di contro Baubom ${ }^{89}$ s'avvidero dell'animale e l'ammazzarono coi remi sì che osso intero non rimase, i marinaj Tonchinesi ${ }^{90}$ lo serbarono per due giorni poscia ne fecero gustosissimo pasto; ci volle la sola autorità del Capitano per salvare quanto Le spedisco. La Nave era l'Unchowa (Italiana) il Capitano Sig.r Rosciano Genovese ${ }^{91}$. I teschi appena che li potrò avere ${ }^{92}$.

Vorrà perdonare se nelle ore notturne precipitai questa mia, non poteva fare a meno, ora non la rileggo per salvarla dalla distruzione o per non arrossire adesso e dappoi, Ella si convincerà che le lettere dei Missionarj amettono errori e strafalcioni in quantità. Nella prima occasione in cui possa incontrarsi coll'Onoratiss. Sig.ra Elisa ${ }^{93}$ Le dica quanto Le potrei io stesso dire di presenza, stringendole la mano a mio carico l'assicuri che già io sul venerato volto del di Lei impareggiabile Padre deposi più di un religioso figliale baccio, entrato per somma mia fortuna nel di Lei Officio trovo alla coscienza conforto di aver soddisfatto quanto non ebbi grazia soddisfare pel mio genitore, Le dica schiettamente che in perpetuo figlia non sarà unica nel rammentarne le virtù di un tanto Padre, e nel dolore mi avrà fratello alla custodia di sì sante reliquie fino a che saranno a Lei passate. Mille ossequj di profondo ed affettuoso rispetto alle Signore De Tavel e Capelli ${ }^{94}$ Sorelle al Defunto, anche a loro un conforto, se un Carissimo Fratello fu Loro involato, oh! non è perduto, che l'Uomo di virtù non muore. Alla Sig.ra Bassi ${ }^{95}$ di cui sì spesso me ne parlava l'Amato De Filippi presenti un mio rispettosissimo saluto e Le attesti la mia stima più sincera. Avrei scritto al Eg. Conte Giulio Casati ${ }^{96}$, la mancanza d'addresso me lo impedì, siccome pure alla detta Signora.

Con Lei Signor Direttore non aggiungo più parole, che io possa trovarle quanto mai un sagace scrutatore può rinvenire, ed Ella con tutta la libertà mi chiami quello che perviene sapere esistente in queste contrade, sarà mia somma premura di fare le spedizioni pronte e colla massima possibile diligenza e con ciò potrò meritarmi la di Lei preziosa relazione.

Permetta che con illimitata stima e profonda riverenza mi rassegni

Di Lei Sig.r Direttore

Obbligatiss. ed Umil Servo

Sac. Bernardo Viganò Miss. Ap.

P.S. Essendomi capitati varj disordini nelle spedizioni e tardanze m'ebbi necessario eleggermi un ricapito ed un ricevente fedele. Questi è il M.R. Preposto di Merate D. Eliseo Bordoni ${ }^{97}$ di cui fui Coadjutore, e tiene casa paterna in Milano presso la Canonica di St. Simpliciano, in caso che non mi capitasse occasione diretta per raccomandarne il ricapito diretto al Museo Civico, Ella tutto riceverà $\left[.^{98}\right]$ da quella $\left[\ldots{ }^{99}\right]$ mentre quel M.R. Preposto riceve tutte le mie corrispondenze.

Il ricapito in Milano è: Sig.r Samuele Bordoni.

Essendovi un Missionario Italiano che rimpatria ed è di Napoli mi valgo di Lui per il sicuro ricapito, servendomi delle sole Poste interne.

\section{Note}

1 Museo del Risorgimento, Civiche Raccolte Storiche del Comune di Milano (d'ora in poi CRSM), Archivio Verga, b. 17, Lettere a Verga C-E, lettera di De Filippi a Verga, s.1. [1864-65]. Ringrazio la dott.ssa Paola Zocchi per la segnalazione della lettera.

2 Più in generale sul viaggio e sulla missione della Magenta si vedano Giglioli E. H., 1868; Arminjon V., 1869; Giglioli E. H., 1875; Ammannati F. \& Calzolari S., 1985; Moro T., 2001; Francioni A., 2003 e la bibliografia ivi segnalata. 
3 Giglioli nacque a Londra da Giuseppe ed Ellen Hillyer. Zoologo, etnografo e antropologo, nel 1869, al ritorno della Magenta, fu nominato professore di Zoologia dei Vertebrati al Regio Istituto di Studi Superiori Pratici e di Perfezionamento di Firenze, dirigendo il Museo di Storia Naturale, dove allestì la Collezione Centrale dei Vertebrati Italiani. Autore tra l'altro dell'Avifauna italica (1886), fu protagonista di numerose campagne naturalistiche, come quella del 1881 per lo studio delle acque profonde del Mediterraneo. Ricoprì numerosi incarichi governativi, tra cui la direzione dell'Ufficio Ornitologico e quella della Commissione Consultiva per la Pesca. Alla morte, la sua ingente collezione etnografica entrò a far parte del Museo Nazionale di Preistoria ed Etnografia di Roma, fondato e diretto dal paletnologo Luigi Pigorini. Cfr. i saggi di vari autori in Enrico Hillyer Giglioli, 1996; Romano M. C., 2007. La corrispondenza di Giglioli è oggi conservata presso il Museo Zoologico "La Specola" di Firenze, ma riguarda il periodo successivo al viaggio della Magenta (1875-1900).

4 Durante la spedizione Biasi si occupò della conservazione degli esemplari zoologici. Originario di Trento, nel 1865 era impiegato come "servente" nel Gabinetto di Storia Naturale dell'Università di Genova diretto da Salvatore Trinchese e Arturo Issel (Celesia E., 1867: 179).

5 Arminjon nacque a Chambéry da Mattia ed Enrichetta Dupuy. Dopo aver frequentato la Scuola di Marina a Genova, partecipò alla campagna di Crimea. Nel 1860, in seguito alla cessione della Savoia, optò per la marina francese, tornando un anno dopo in quella italiana, dove intraprese una brillante carriera. Al ritorno della Magenta fu promosso capitano di vascello e nel 1876 contrammiraglio. Collaboratore della Rassegna Nazionale e della Rivista Marittima, fece parte fino alla morte del Consiglio Comunale di Genova, tra le fila dei gruppi cattolici. Sul suo fondamentale ruolo durante la missione della Magenta, cfr. Francioni A., 2003.

6 Cfr. Biblioteca Museo Civico Storia Naturale di Milano (d'ora in poi Biblioteca MSNM), b. 2, fasc. 42a, lettere di De Filippi a Cornalia e Archivio Centrale dello Stato (d'ora in poi ACS), Fondo Negri, b. 2, fasc. 105, lettera di De Filippi a Negri, Napoli 7 novembre 1865.

7 BibliotecaMSNM,Fondo Cornalia, b. 2, fasc.42, letteradiDeFilippia Cornalia, Torino 8ottobre 1865.

8 Biblioteca MSNM, Fondo Cornalia, b. 19, fasc. 6, Cornalia, Alcune parole su Filippo De Filippi.

9 Biblioteca MSNM, Fondo Cornalia, b. 2, fasc. 42, lettera di De Filippi a Cornalia, 19 ottobre 1865.

10 Murray fu in servizio a Hong Kong dal 1858 al 1872, anno in cui fece ritorno in Inghilterra. Di origini scozzesi, studiò medicina all'Università di Edimburgo, dove conobbe il padre di Giglioli, Giuseppe, medico seguace di Mazzini, esule dall'Italia per motivi politici. Interessato alle scienze naturali, accompagnò Giglioli e De Filippi nelle loro visite all'ospedale e al penitenziario di Hong Kong, cfr. Giglioli E. H., 1875: 663, 673; per il necrologio cfr. John Ivor Murray, 1903.

11 Cfr. in particolare De Filippi F., 1855; De Filippi F., 1859, ma anche De Filippi F., 1854. Sulla sua posizione rispetto alla teoria dell'evoluzione, cfr. Benasso G., 1976: 56-68; Pancaldi G., 1983: 137.

12 In Camerano L., 1896: 356; anche in Giacobini G. \& Panattoni G. L., 1983: 16-17. Sulla vita e sulle opere di De Filippi cfr. Lessona M., 1867; Moleschott J., 1867; Omboni G., 1867; Landucci G., 1996 e la bibliografia ivi segnalata.

13 Biblioteca MSNM, Fondo Cornalia, b. 4, fasc. 13, lettera di Lessona a Cornalia, Genova $1^{\circ}$ febbraio 1864.

14 CRSM, Archivio Cattaneo, Cart. 3, pl. XXVII, n. 69, lettera di Cattaneo a Woodcock, Sat.[urday] 30 1867. Per la traduzione in italiano si veda Cattaneo C., 1956: 508-509. L'attribuzione del 30 settembre 1867 come data della lettera è erronea. Si tratta infatti di sabato 30 marzo 1867. Ringrazio la dott.ssa Mariachiara Fugazza per la segnalazione del documento.

15 Cfr. Archivio Storico Civico di Milano e Biblioteca Trivulziana (d'ora in poi ASCM), Registri di matrimonio 1842, Milano parrocchia San Fedele, 3 luglio 1842; Barbieri G., 1846.

16 Giglioli E. H., 1875: 96; sul ferimento di Giglioli cfr. Biblioteca MSNM, Fondo Cornalia, b. 2, fasc. 42a, lettera di De Filippi a Cornalia, A bordo della Magenta prima della discesa a Saigon $1^{\circ}$ giugno 1866.

17 Cfr. Giglioli E. H., 1875: 661-696. In una lettera a Cornalia del 25 maggio 1867 da Melbourne, Giglioli forniva informazioni simili, Biblioteca MSNM, Fondo Cornalia, b. 3, fasc. 35.

18 Dent era a capo della Dent \& Company, una delle più antiche e fiorenti case di commercio inglesi presenti in Cina per quanto riguardava la seta, il tè e l'oppio, finita in bancarotta tra il $1866 \mathrm{e}$ il 1867. Aveva assunto la carica di console per il Re di Sardegna a Hong Kong nel 1858, rappresentando in seguito anche il Regno d'Italia.

19 Per alcuni cenni biografici cfr. Bernardo Viganò, 1901; sulla storia del PIME in quegli anni, cfr. Tragella G. B., 1959. Ringrazio la dott.ssa Laura Denaro per le segnalazioni riguardanti Viganò e per la ricerca iconografica nell'Archivio fotografico del PIME.

20 Cfr. Biblioteca MSNM, Fondo Cornalia, b. 8, fasc. 13, lettera di Viganò a Cornalia, Isole FilippineManila $1^{\circ}$ febbraio 1869. 
21 Cfr. Giglioli E. H., 1875: 726. Il 3 luglio 1867 il primo console italiano in Giappone, Cristoforo Robecchi, riferiva a Cornalia che John Dent si era lamentato per non aver ricevuto indicazioni riguardo al monumento funebre in onore di De Filippi, cfr. Biblioteca MSNM, Fondo Cornalia, b. 6, fasc. 15. Nel 1868 nel loggiato del Palazzo dell'Università di Torino fu inaugurato un busto in ricordo dello zoologo. Per le cerimonie del 1879 a Pisa si vedano i due giornali locali: Corriere dell'Arno, 14 settembre 1879 e La Croce Pisana, 13 settembre 1879.

22 Per la lettera di Viganò, cfr. Biblioteca MSNM, Fondo Cornalia, b. 6, fasc. 14, doc. 25; per il fascicolo intestato al solo missionario, cfr. b. 8, fasc. 13. Per informazioni su queste figure, cfr. Ciapparoni La Rocca T., 2001; Ciapparoni La Rocca T., 2007; Fiussello N., 2007; Zanier C., 2006.

23 Cfr. Biblioteca MSNM, Fondo Cornalia, b. 6, fasc. 12, lettera di Richiardi a Cornalia, Bologna 28 marzo 1867.

24 Per la disponibilità durante le ricerche, ringrazio la dott.ssa Paola Livi, Biblioteca del Museo di Storia Naturale di Milano, la dott.ssa Paola Novaria, Archivio Storico dell'Università di Torino, il dott. Marco Moietta, Biblioteca del Dipartimento di Biologia, e i loro collaboratori.

25 Semaio e imprenditore serico di Milano collegato al commercio internazionale del seme bachi, nel 1863 Meazza fu protagonista, insieme a Pompeo Litta e Modesto Gavazzi, della sfortunata spedizione a Bukhara, città dell'Uzbekistan, durante la quale fu imprigionato per un anno insieme ai compagni con l'accusa di spionaggio. Nel marzo 1867 iniziarono i suoi numerosi viaggi verso il Giappone per scopi commerciali. Fu anche un fine collezionista d'arte. Enrico Cernuschi, milanese esule a Parigi dopo il 1848, nel 1875 comprò da Meazza una preziosa collezione di porcellane e ceramiche giapponesi, oggi conservata al Museo Cernuschi di Parigi. Oggetti orientali a lui appartenuti furono acquistati nel 1910 anche dalle Raccolte d'Arte Applicata del Castello Sforzesco di Milano. Cfr. Beretta L., 2001; Zanier C., 2006; Zanier C., 2008.

26 Biblioteca MSNM, Fondo Cornalia, b. 5, fasc. 16, doc. 94, lettera di Panceri a Cornalia, s.1. s.d. Panceri proseguiva criticando l'operato di Giglioli: "Mi fa stupore che non si parli di Giglioli, forse che partendo la Magenta egli pure è partito abbandonandolo. Sarebbe stata dura, né io mi meraviglierei poiché credo che Giglioli non abbia cuore alcuno. Io assistei alla sua partenza, poiché come sai facemmo viaggio insieme da Firenze a Napoli, e il modo freddo con cui Giglioli lasciò la madre mi gelò il sangue".

27 Cfr. Biblioteca MSNM, Fondo Cornalia, b. 6, fasc. 31, lettera di Salvadori a Cornalia, Torino 18 maggio 1867; b. 6, fasc. 51, lettera di Lessona a Cornalia, 8 ottobre 1867. La conferenza era annunciata sulla Gazzetta Piemontese del 19 maggio 1867. Sull'interessante figura di Lessona, traduttore di Darwin, tra i maggiori divulgatori scientifici dell'Italia della seconda metà Ottocento, cfr. da ultimo Govoni P., 2002: 165-206; Scaringella A., 2011.

28 Cfr. Biblioteca MSNM, Fondo Cornalia, b. 4, fasc. 54, lettera di Moleschott a Cornalia, Torino 18 maggio 1867; Moleschott J., 1867.

29 Cfr. Atti della Società Italiana di Scienze naturali 1868, 11: 359. Cornalia tenne il suo discorso il 17 settembre nella seduta generale di chiusura presieduta dal naturalista Paolo Lioy.

30 Biblioteca MSNM, Fondo Cornalia, b. 19, fasc. 6, Cornalia, Alcune parole su Filippo De Filippi.

31 Biblioteca MSNM, Fondo Cornalia, b. 2, fasc. 42a, lettera di De Filippi a Cornalia, Hong Kong 29 dicembre 1866.

32 Biblioteca MSNM, Fondo Cornalia, b. 2, fasc. 42a, lettera di De Filippi a Cornalia, Pekino 21 ottobre 1866. De Filippi motivava così il suo giudizio: "Non abbiamo buoni cronometri, non abbiamo sonde di grande profondità, non abbiamo neppure termometri per misurar la temperatura dell'acqua". Per questo la parte zoologica sarebbe stata a suo parere quella più ricca di risultati.

33 Sul rapporto tra Chiesa ed evoluzionismo, cfr. Artigas M., Glick T. F. \& Martínez R. A., 2008; De Lauri A., 2010.

34 Lessona M., 1867: 654. Su questo punto gli faceva eco anche Cornalia nel discorso di Vicenza dedicato a De Filippi. In una lettera di Antonio Gigli a Giambattista Benasso dell'11 ottobre 1982 si apprende che al momento della morte lo zoologo stava leggendo il libro di Angelo Fava, Fede e Ragione. Guida alla dottrina religiosa pei giovani studenti di filosofia (1851). Ringrazio l'erede di De Filippi, prof. Guido Gigli, per la preziosa collaborazione e per avere messo a disposizione quanto emerso dalle ricerche effettuate da suo padre Antonio e quanto si è conservato dell' archivio De Filippi. Ringrazio inoltre il dott. Dario Moretta per avermi aiutata a rintracciare gli eredi.

35 Cfr. ASCM, Ruolo generale di popolazione 1835, v. 22.

36 Archivio Storico dell'Università di Torino, Affari ordinati per classe, 1867, fasc. 8.4 Museo di Zoologia e Anatomia Comparata, XIV B 13 (d'ora in poi ASUT, 8.4, XIV B 13), Testamento De Filippi, Hong Kong 2 febbraio 1867. 
37 Fulvio Piacenza nacque a Milano il 12 dicembre 1842 da Andrea e Barbara Gerli. Aveva una sorella, Caterina, nata a Milano nel 1839, cfr. ASCM, Ruolo generale di popolazione 1835, v. 44.

38 Cfr. Biblioteca MSNM, Fondo Cornalia, b. 19, fasc. 6, Cornalia, Alcune parole su Filippo De Filippi.

39 Biblioteca MSNM, Fondo Cornalia, b. 5, fasc. 16, lettera di Panceri a Cornalia, 17 luglio 1867.

40 Biblioteca MSNM, Fondo Cornalia, b. 6, fasc. 12, lettera di Richiardi a Cornalia, Bologna 28 marzo 1867.

41 Biblioteca MSNM, Fondo Cornalia, b. 6, fasc. 12, doc. 38, lettera di Richiardi a Cornalia, s.l. s.d.

42 Cfr. ACS, M.P.I. Personale 1860-1880, b. 1163, fasc. Michele Lessona, lettera di Sella [al Ministro Pubblica Istruzione], s.l. s.d.

43 Biblioteca MSNM, Fondo Cornalia, b. 6, fasc. 12, doc. 38, lettera di Richiardi a Cornalia, s.1. s.d.

44 Ancora nel 1874, Richiardi reclamava, ottenendola, la restituzione di circa 300 lire che De Filippi aveva consegnato a Giglioli durante la spedizione della Magenta, cfr. Biblioteca Dipartimento di Biologia Animale e dell'Uomo dell'Università di Torino (d'ora in poi Biblioteca Storica DBAU), Quaderni Copialettere di Lessona, 41 G 10, minute di Lessona a Richiardi, 9 e 24 febbraio 1874.

45 Cfr. Biblioteca Storica DBAU, Carte De Filippi, Atto di notificanza della Pretura di Torino, 4 maggio 1867.

46 Cfr. ASUT, 8.4, XIV B 13, lettera di Salvadori al Rettore, Torino $1^{\circ}$ giugno 1867.

47 ASUT, 8.4, XIV B 13, lettera del Ministro al Rettore, Firenze 18 settembre 1867. Questo documento, come molti altri del fascicolo, è firmato dal Segretario Generale del Ministero, Federico Napoli (1811-1908), in un momento di veloce avvidendamento di ministri.

48 ASUT, Affari ordinati per classe, 1868-1869, fasc. 8.4 Museo di Zoologia e Anatomia Comparata, XIV B 20 (d'ora in poi ASUT, 8.4, XIV B 20), lettera del Ministro al Rettore, Firenze 9 gennaio 1869.

49 Cfr. ASUT, 8.4, XIV B 20, minuta del Rettore al Ministro, Torino 4 marzo 1869.

50 Biblioteca MSNM, Fondo Cornalia, b. 6, fasc. 12, lettera di Richiardi a Cornalia, Bologna 5 aprile 1869. Allo stato attuale della ricerca, non si hanno notizie di dove si trovi la biblioteca De Filippi.

51 Cfr. Biblioteca Storica DBAU, Carte De Filippi, lettera del Procuratore Generale al Direttore del Museo Zoologico, Torino 15 marzo 1868. Altre 22 casse, arrivate a Genova il 10 aprile 1868, erano state spedite da Giglioli, appena sbarcato a Napoli, al ritorno della Magenta: due erano destinate al Museo Industriale; le rimanenti contenevano esemplari naturalistici raccolti dopo la morte di De Filippi per il Museo Zoologico, cfr. ASUT, 8.4, XIV B 20, lettera di Lessona al Rettore, 9 aprile 1868. Codazza si lamentò con Lessona di aver ricevuto solo due casse, oltretutto quasi vuote, e non quattro come annunciava De Filippi nel testamento. Sulla vicenda cfr. Biblioteca Storica DBAU, Carte De Filippi, lettera di Codazza a Lessona, Torino 18 aprile 1868 e ASUT, 8.4, XIV B 20, lettera del Ministro al Rettore, Firenze 25 aprile 1868.

52 ASUT, 8.4, XIV B 20, lettera del Ministro al Rettore, Firenze 19 aprile 1869.

53 ASUT, 8.4, XIV B 20, lettera del Ministro al Rettore, Firenze 22 marzo 1869.

54 Cfr. Biblioteca Storica DBAU, Carte De Filippi, lettera del Ministro al Rettore, 2 maggio 1868.

55 ASUT, 8.4, XIV B 20, lettera di Villari al Rettore, Firenze 13 settembre 1869.

56 Cfr. ASUT, 8.4, XIV B 20, lettera del Ministro al Rettore, 11 ottobre 1869; minuta del Rettore a Codazza e Battisti, 14 ottobre 1869; minuta del Rettore al Ministro, 30 ottobre 1869.

57 Biblioteca Storica DBAU, Carte De Filippi, lettera del Ministro al Rettore, 2 maggio 1868.

58 Biblioteca MSNM, Fondo Cornalia, b. 5, fasc. 16, doc. 88, lettera di Panceri a Cornalia, s.1., s.d.

59 Biblioteca MSNM, Fondo Cornalia, b. 19, fasc. 6, Cornalia, Alcune parole su Filippo De Filippi.

60 Biblioteca MSNM, Fondo Cornalia, b. 6, fasc. 12, lettera di Richiardi a Cornalia, Bologna 5 aprile 1869. Nelle Carte Richiardi conservate presso la Biblioteca del Dipartimento di Scienze Naturali e Ambientali dell'Università di Pisa non sono presenti i taccuini e gli appunti di De Filippi. Dalla già citata lettera di Antonio Gigli a Benasso si apprende che questi materiali andarono distrutti durante la seconda guerra mondiale. Nello stesso Dipartimento è invece depositata la Biblioteca Richiardi, composta da numerose miscellanee di argomento naturalistico.

61 Cfr. le lettere da Rio de Janeiro del 6 gennaio 1866, da Batavia del 27 aprile 1866 e da Hong Kong del 10 gennaio 1867, in Atti della R. Accademia delle Scienze di Torino 1866, 1: 375-390; 601-610; 1867, 2: 227-238.

62 Cfr. Lessona M., 1867: 654-659. Alla fine della commemorazione, parlando della morte di De Filippi, Lessona riportava alcune notizie molto simili a quelle fornite da Viganò nella lettera a Cornalia. 
63 ACS, Fondo Negri, b. 2, fasc. 105, lettera di De Filippi a Negri, Mar Giallo a bordo della Magenta 11 novembre 1866. Nella stessa lettera De Filippi gli annunciava l'invio della traduzione dal francese all'italiano da lui eseguita del diario di viaggio di un cinese in Cocincina, come allora veniva chiamata la regione più meridionale del Vietnam. La versione originale cinese era stata tradotta dall'interprete e console onorario francese a Pechino, Henri Fontanier, che aveva dato a De Filippi il permesso di renderla in italiano. Il testo così tradotto fu poi pubblicato nel Bollettino della Società Geografica Italiana, cfr. Giornale d'un ambasciatore chinese, 1868.

64 Angela (o Angiola) De Filippi, detta Nina, sorella di Filippo, nata a Milano nel 1812, cfr. ASCM, Ruolo generale di popolazione 1835, v. 22. Sposata in prime nozze con Paolo Vanotti e in seconde nozze con Franz-Carl von Tavel, uomo politico di Berna, presidente dell'Assemblea dei deputati dei cantoni svizzeri nel 1835. Fu spesso a Parigi, dove fu in contatto con la scrittrice francese Marie Catherine Sophie, contessa D'Agoult, celebre per il salotto parigino, che a partire dal 1848 rappresentò un influente centro di azione politica. Nell'Archivio Cattaneo è conservato un breve scambio epistolare risalente al 1859, di tono confidenziale e patriottico, tra Cattaneo, Nina Vanotti e la figlia Jenny Collino Vanotti, quando queste risiedevano a Luvinate, presso Varese, cfr. CRSM, Archivio Cattaneo, Cart. 2, pl. 19, n. 33; Cart. 3, pl. 30, n. 53; Cart. 7, pl. 25, n. 30, n. 40.

65 L'imprenditore serico milanese Meazza, diretto a Yokohama, incontrò Viganò durante il breve scalo a Hong Kong. In quell'occasione cercò senza successo di far rientrare di contrabbando in Italia la salma di De Filippi, per evitare le difficoltà e i costi del trasporto di resti umani sulle linee di navigazione delle Messageries Imperiales francesi. Cfr. Biblioteca MSNM, Fondo Cornalia, b. 4, fasc. 44, lettera di Meazza a Cornalia, a bordo del Duplex sul Han Kiang vicino Shang-ha 3 giugno 1867.

66 La Magenta salpò il 18 gennaio.

67 La Magenta rientrò in porto il 20 gennaio.

68 Vittorio Arminjon.

69 Camillo Cucca (1829-1893), di Napoli, era l'ufficiale medico della Magenta. Morì per cause di servizio in seguito a una missione sanitaria.

70 Tra i medici interpellati dal comandante Arminjon, oltre a John Ivor Murray e Cucca, Giglioli menzionava nella sua relazione anche Robert Bernard, medico capo della marina inglese, che assistette De Filippi nelle ultime fasi della malattia, Henry Dick, medico capo del presidio, e Joseph Beale, chirurgo in servizio sulla fregata americana Hartford. Cfr. Giglioli E. H., 1875: 696.

71 Il 26 gennaio la Magenta riprese il suo viaggio diretta in Australia con a bordo sia Giglioli che Biasi.

72 Richard Graves MacDonnell (1814-1881) fu il governatore di Hong Kong dal 1866 al 1872, dopo aver ricoperto ruoli analoghi in altri possedimenti dell'Impero britannico.

73 John Dent.

74 L'Hotel d'Europe era situato in Hollywood Road, una delle strade principali nel quartiere centrale di Hong Kong. In una guida del tempo era definito "the best establishment, [...] frequently overcrowded on the arrival of the mail steamers from Europe or the North", Dennys N. B., 1867: 23.

75 De Filippi fu sbarcato il 24 gennaio.

76 Sinonimo di tisi.

77 Si tratta del Regio Museo Industriale Italiano di Torino.

78 Ministero di Agricoltura, Industria e Commercio, da cui il Museo Industriale dipendeva.

79 Murray assistette De Filippi fino al 23 febbraio. Nella fase finale della malattia lo zoologo italiano fu affidato alle cure di Robert Bernard e Richard Alfred O’Brien, cfr. Giglioli E. H., 1875: 726.

80 Nel carteggio Cornalia del Museo Civico milanese non sono conservate lettere di Nina De Tavel.

81 Alla cerimonia funebre parteciparono i consoli europei e i rappresentanti del governatore, della comunità europea di Hong Kong e della marina inglese e americana. Si veda la Gazzetta Ufficiale del Regno d'Italia del 26 marzo 1867; Giglioli E. H., 1875: 726.

82 Alessandro Ripamonti (1815-1872), ordinato sacerdote nel 1838, entrò nel Seminario Lombardo delle Missioni Estere fin dalla sua fondazione, nel 1850. Ne fu il primo economo generale a vita. Cfr. il necrologio Alessandro Ripamonti, 1872.

83 A quel tempo il Museo Civico di Storia Naturale possedeva una raccolta antropologica voluta da Cornalia, ricca di teschi, alcuni in gesso. Essa era formata dalla raccolta di anatomia comparata, a cui si erano aggiunte la raccolta frenologica donata da Giovanni Fossati nel 1864, 50 maschere in gesso donate nel 1865 dal geografo Adolf Schlagenweit e numerosi crani egiziani offerti dall'egittologo Luigi Vassalli nel 1867. I cataloghi di queste raccolte sono andati dispersi, cfr. Livi P., 2008.

84 John Ivor Murray. 
85 I quattro teschi di cinesi procurati da Murray furono effettivamente inviati al Museo Industriale di Torino, come dimostra la lettera del 23 luglio 1867 dell'assistente al Consolato italiano a Hong Kong N. J. Ede al direttore di quel Museo. Si trattava dei teschi di quattro pirati originari della Cina meridionale, da poco impiccati a Hong Kong, cfr. Biblioteca Storica DBAU, Carte De Filippi. Nel 1875 Giglioli riferiva che i crani erano conservati presso il Museo Nazionale di Antropologia di Firenze diretto da Paolo Mantegazza, Giglioli E. H., 1875: 674.

86 Potrebbe trattarsi della lettera che Cornalia aveva spedito all'amico il 18 settembre 1866, a cui De Filippi accennava nella sua ultima comunicazione del 29 dicembre 1866, dove riferiva a Cornalia di non aver potuto raccogliere ancora nulla per lui, cfr. Biblioteca MSNM, Fondo Cornalia, b. 2, fasc. $42 \mathrm{a}$.

87 Si tratta molto probabilmente di un rettile della specie Physignathus cocincinus, molto diffuso in Tailandia, Cambogia, Indocina e nel Vietnam meridionale, comunemente chiamato drago d'acqua cinese. Ringrazio il dott. Stefano Scali, erpetologo del Museo Civico di Storia Naturale di Milano, per l'individuazione della specie. Forse potrebbe trattarsi dell'esemplare a secco di Varanus proveniente da Hong Kong, donato al Museo il 18 dicembre 1867 da Ferdinando Meazza, come segnalato nell'Elenco dei donatori 1847-in avanti, in Biblioteca MSNM.

88 La Cocincina è la regione storica del Vietnam, corrispondente alla sezione più meridionale del paese.

89 Città marittima dell'allora Impero dell'Annam, regione dell'Indocina costituita dalla fascia costiera orientale del Vietnam.

90 Provenienti da Tonchino, la parte più settentrionale del Vietnam.

91 Potrebbe trattarsi di Giacomo Galleano Rosciano (1820-1898), nato a Celle ligure, vicino Genova, che conseguì la patente di lungo corso, navigando in molte parti del mondo, cfr. Bruzzone G. L., 2006.

92 In una lettera da Yokohama del 14 luglio 1867, Meazza informava Cornalia di aver chiesto a Viganò almeno una dozzina di teschi (Biblioteca MSNM, Fondo Cornalia, b. 4, fasc. 44). Da una lettera del 3 gennaio 1869 di Viganò a Cornalia si apprende però che il missionario non li aveva ancora inviati, anche se sperava di farlo presto, cfr. Biblioteca MSNM, Fondo Cornalia, b. 8, fasc. 13. Nell'Elenco dei donatori 1847-in avanti non risulta l'arrivo dei teschi in Museo.

93 Elisa Richiardi De Filippi.

94 Giuseppa (o Giuseppina) De Filippi, sorella di Filippo, nata a Milano nel 1816 e sposata con Antonio Capelli (1803-1874), medico patriota compromesso nelle vicende politiche del 1848, docente dal 1835 ad almeno il 1869 all'Imperial Regio Istituto Veterinario di Milano (poi Regia Scuola Superiore di Medicina Veterinaria), dove insegnò varie materie, dalla chimica alla patologia generale e alla zootomia patologica. Cfr. ASCM, Ruolo generale di popolazione 1835, v. 22; Archivio di Stato di Milano, Ufficio del registro successioni, Cartella 59, Antonio Capelli.

95 Virginia Olivazzi (1814-1882), figlia di Francesco e Giulia Carlovitz, moglie del nobile Carlo Bassi, entomologo e uomo politico, che dal 1841 al 1856, anno della morte, fece parte del Collegio dei Conservatori del Museo Civico di Storia Naturale di Milano e che nel 1844 ricoprì la carica di Segretario Generale alla Sesta Riunione degli Scienziati Italiani che si tenne a Milano. Un anno dopo la morte di Bassi, Virginia, assecondando le volontà del marito, donò al Museo i suoi libri scientifici e la sua collezione di coleotteri europei. Il nome di Virginia ricorre in molti dei carteggi dei naturalisti che gravitarono in quegli anni intorno al Museo di Storia Naturale di Milano, come Cornalia, De Filippi e Panceri.

96 Si tratta con ogni probabilità di Gabrio Casati (1798-1873), podestà di Milano dal 1837 al 1848 , anni in cui seguì in prima persona la fondazione del Museo Civico di Storia Naturale. Si trasferì in Piemonte nel 1848, dove fu nominato senatore nel 1853. Ministro della Pubblica Istruzione dal luglio 1859 al gennaio 1860, elaborò la legge, detta appunto legge Casati, che regolò la politica scolastica italiana fino alla riforma Gentile del 1923. Fu vicepresidente e poi presidente del Senato dal 1865 al 1872, dove, il 31 marzo 1867, rese omaggio alla figura di De Filippi, si vedano i Rendiconti del Parlamento italiano. Discussioni del Senato del Regno, tornata del 31 marzo 1867, 1: 10. Nel 1868, come si evince da una lettera a Cornalia, Casati s'interessò personalmente delle vicende riguardanti l'eredità De Filippi, cfr. Biblioteca MSNM, Fondo Cornalia, b. 2, fasc. 11, lettera di Casati a Cornalia, 17/1868.

97 Eliseo Bordoni (1800-1870), professore di Belle Lettere nei Seminari Diocesani e Rettore del Seminario di Monza. Fu parroco a Merate dal 1843 alla morte.

98 Parola illeggibile.

99 Illeggibile a causa del foglio strappato. 


\section{Bibliografia}

Alessandro Ripamonti [necrologio], 1872 - Le Missioni Cattoliche, Milano, 23 novembre 1872, (34): 404-405.

Ammannati F. \& Calzolari S., 1985 - Un viaggio ai confini del mondo 1865-1868. La crociera della pirocorvetta Magenta dai documenti dell'Istituto Geografico Militare. Sansoni, Firenze.

Arminjon V., 1869 - Il Giappone e il viaggio della corvetta Magenta nel 1866. Tipi del R.I. De'Sordomuti, Genova.

Artigas M., Glick T. F. \& Martínez R. A., 2008 - Darwin and the Vatican: the Reception of Evolutionary Theories. In: The Reception of Charles Darwin in Europe. Engels E. M. \& Glick T. F. (eds.). Continuum, London-New York, 2: 413-429.

Barbieri G., 1846 - Benedizione di nozze per li signori Dottore Filippo De Filippi e Angelina Vallardi di Milano. In: Avvento predicato in San Fedele a Milano l'anno 1843 con altri discorsi. s.n., Italia: 367-372.

Benasso G., 1976 - Da Bonelli a De Filippi (1811-1864). Materiali per una storia dell'evoluzionismo italiano. Atti della Accademia Roveretana degli Agiati, Rovereto, 14-15: 3-106.

Beretta L., 2001 - Ferdinando Meazza avventuroso collezionista milanese. Rassegna di studi e di notizie, Milano, 25: 143-163.

Bernardo Viganò [necrologio], 1901 - Le Missioni Cattoliche, Milano, 12 luglio 1901, (28): 336.

Bruzzone G. L., 2006 - Lettere di Giuseppe Garibaldi a Giacomo Galleano Rosciano. Rassegna Storica del Risorgimento, Roma, 93 (2): 263-270.

Camerano L., 1896 - La vita scientifica di Michele Lessona. Commemorazione letta alla classe di scienze fisiche, matematiche e naturali della R. Accademia di Torino il dì 17 novembre 1895. Memorie della Reale Accademia delle Scienze di Torino, Torino, 45: 331-388.

Cattaneo C., 1956 - Epistolario di Carlo Cattaneo. Caddeo R. (a cura di). Barbera, Firenze, v. 4.

Celesia E., 1867 - Storia dell’Università di Genova dal 1814 fino a dì nostri. Tip. Sordomuti, Genova.

Ciapparoni La Rocca T., 2001 - I diari giapponesi di M.Me Sallier De La Tour. In Atti del XXV Convegno di Studi Giapponesi. Aistugia, Venezia: 191-202.

Ciapparoni La Rocca T., 2007 - I viaggi di Pietro Savio (1838-1904). In: Italiani nel Giappone Meiji (1868-1912). Atti del Convegno Internazionale Bilaterale. Ciapparoni La Rocca T., Fedi P. \& Lucidi M. T. (a cura di). Centro Stampa Università, Roma: 96-105.

De Filippi F. 1854 - La creazione terrestre. Lettere a mia figlia. Dell'Antica Ditta Pietro e Giuseppe Vallardi, Milano.

De Filippi F., 1855 - Il diluvio noetico. Tip. Scolastica di Sebastiano Franco e Figli, Torino. Estratto da Cimento, Torino, 6 (2).

De Filippi F. 1859 - L'uomo e il diluvio. Lettera al sig. E. Littré. Rivista Contemporanea. Torino, 19: 76-96.

De Filippi F., 1864 - L'uomo e le scimie. Lezione pubblica detta in Torino la sera dell'11 gennaio 1864. Il Politecnico, Milano, 21: 5-32. 1865, G. Daelli, Milano.

De Lauri A., 2010 - La "patria" e la "scimmia". Il dibattito sul darwinismo in Italia dopo l'Unità. Biblion Edizioni, Milano.

Dennys N. B. 1867 - The Treaty Ports of China and Japan. Trübner and Co.-Shortrede and Co., London-Hong Kong. 
Enrico Hillyer Giglioli: l'uomo, il naturalista, il viaggiatore, 1996 - L’Universo, Firenze, 76 (5): 625-672.

Fiussello N., 2007 - Cristoforo Robecchi, Yokohama 1867-1871. Gli esiti culturali di un incarico diplomatico in Giappone. In: Italiani nel Giappone Meiji (18681912). Atti del Convegno Internazionale Bilaterale. Ciapparoni La Rocca T., Fedi P. \& Lucidi M. T. (a cura di). Centro Stampa Università, Roma: 73-80.

Francioni A., 2003 - Il trattato italo-cinese del 1866 nelle carte dell'ammiraglio Arminjon. Università degli Studi di Siena, Siena.

Giacobini G. \& Panattoni G. L., 1983 - Il darwinismo in Italia. Unione Tipografico-Editrice Torinese, Torino.

Giglioli E. H., 1868 - Cenni generali sul viaggio di circumnavigazione della pirocorvetta Magenta 1865-66-67-68. Bollettino della Società Geografica Italiana, Firenze, 1 (1): 215-241.

Giglioli E. H., 1875 - Viaggio intorno al globo della R. Pirocorvetta italiana Magenta. Maisner Editori, Milano.

Giornale d'un Ambasciatore chinese spedito in Cocincina dall'Imperatore TaoKwang (1840-41), 1868 - Bollettino della Società Geografica Italiana, Firenze, 1 (1): 277-294.

Govoni P., 2002 - Un pubblico per la scienza. La divulgazione scientifica nell'Italia in formazione. Carocci, Roma.

John Ivor Murray [necrologio], 1903 - The British Medical Journal, London, 2 (2223): 339-340.

Landucci G., 1996 - De Filippi Filippo. In: Dictionnaire du Darwinisme et de l'Évolution. Tort P. (a cura di). Presses Universitaires de France, Paris, 1: 11341139.

Lessona M., 1867 - Filippo De Filippi. Nuova Antologia, Firenze, 6 (12): 631-660.

Livi P., 2008 - La storia naturale dell'uomo nella Milano dell'Ottocento. Un viaggio attraverso le raccolte del Museo Civico di Storia Naturale. Atti della Società Italiana di Scienze Naturali e del Museo civico di Storia Naturale in Milano, Milano, 149 (2): 273-292.

Macchi M., 1867 - Non è possibile. Il Libero Pensiero. Giornale dei Razionalisti, Milano, 2 (14): 221-222.

Moccagatta L., 1867 - Ultimi momenti del Senatore De Filippi in Hong Kong. Museo delle Missioni Cattoliche, Torino: 274-276.

Moleschott J., 1867 - Cenno biografico sul socio Prof. Filippo De Filippi. Atti della R. Accademia delle Scienze di Torino, Torino, 2 (5): 431-453.

Moro T., 2001 - Le campagne oceaniche della Regia Marina nei primi decenni dell'Unità e la conclusione di trattati con Cina, Giappone e Siam. In: Missioni militari italiane all'estero in tempo di pace (1861-1939). Atti del Convegno di Studi. Rainero R. H. \& Alberini P. (a cura di). Commissione Italiana di Storia Militare, Roma: 11-81.

Morte del senatore De Filippi, 1867 - L'Osservatore Cattolico, Milano, 26 marzo 1867, (70): 278-279.

Negri C., 1870 - Il viaggio della «Magenta». Bollettino della Società Geografica Italiana, Firenze, 3 (4): 258-60.

Omboni G., 1867 - Filippo De Filippi. L’Emporio Pittoresco, Milano, 4 (140): 273-275.

Pancaldi G., 1983 - Darwin in Italia. Impresa scientifica e frontiere culturali. Il Mulino, Bologna. 
Romano M. C., 2007 - L'esperienza giapponese di Enrico Hillyer Giglioli: il piacere della scoperta fra collezionismo erudito e gusto per la ricerca. In: Italiani nel Giappone Meiji (1868-1912). Atti del Convegno Internazionale Bilaterale. Ciapparoni La Rocca T., Fedi P. \& Lucidi M. T. (a cura di). Centro Stampa Università, Roma: 65-72.

Scaringella A., 2011 - Michele Lessona. Scienziato di molte arti. Museo Regionale di Scienze Naturali, Torino.

Stefanoni L., 1868 - Sulla morte di Filippo De Filippi. Il Libero Pensiero. Giornale dei Razionalisti, Milano, 3 (2): 21-24.

Tragella G. B., 1959 - Le Missioni estere di Milano nel quadro degli avvenimenti contemporanei, PIME, Milano, v. 2.

Zanier C., 2006 - Semai. Setaioli italiani in Giappone (1861-1880). CLEUP, Padova.

Zanier C., 2008 - Setaioli italiani in Asia. CLEUP, Padova.

\section{Fonti archivistiche}

Archivio Centrale dello Stato (ACS). Fondo Negri; M.P.I. Personale 1860-1880.

Archivio di Stato di Milano (ASM). Ufficio del registro successioni di Milano; Dichiarazioni di successione.

Archivio Storico Civico di Milano e Biblioteca Trivulziana (ASCM). Ruolo generale di popolazione 1835, v. 22, 44; Registri di matrimonio 1842.

Archivio Storico dell'Università di Torino (ASUT). Affari ordinati per classe, 1867, fasc. 8.4 Museo di Zoologia e Anatomia Comparata, XIV B 13 e 1868-1869, XIV B 20.

Dipartimento di Biologia Animale e dell'Uomo dell'Università di Torino, Biblioteca (Biblioteca storica DBAU). Carte De Filippi; Quaderni copialettere di Lessona.

Dipartimento di Scienze Naturali e Ambientali dell'Università di Pisa, Biblioteca (Biblioteca DSNA). Carte Richiardi; Miscellanea Richiardi.

Museo Civico Storia Naturale di Milano, Biblioteca (Biblioteca MSNM). Fondo Cornalia.

Museo del Risorgimento, Civiche Raccolte Storiche del Comune di Milano (CRSM). Archivio Cattaneo; Archivio Verga.

Ricevuto: 2 febbraio 2012

Approvato: 5 marzo 2012 